\title{
Towards the minimal seesaw model via CP violation of neutrinos
}

\author{
Yusuke Shimizu, ${ }^{a}$ Kenta Takagi $^{a}$ and Morimitsu Tanimoto ${ }^{b}$ \\ ${ }^{a}$ Graduate School of Science, Hiroshima University, \\ Higashi-Hiroshima 739-8526, Japan \\ ${ }^{b}$ Department of Physics, Niigata University, \\ Niigata 950-2181, Japan \\ E-mail: yu-shimizu@hiroshima-u.ac.jp, takagi-kenta@hiroshima-u.ac.jp, \\ tanimoto@muse.sc.niigata-u.ac.jp
}

ABSTRACT: We study the minimal seesaw model, where two right-handed Majorana neutrinos are introduced, focusing on the $\mathrm{CP}$ violating phase. In addition, we take the trimaximal mixing pattern for the neutrino flavor where the charged lepton mass matrix is diagonal. Owing to this symmetric framework, the $3 \times 2$ Dirac neutrino mass matrix is given in terms of a few parameters. It is found that the observation of the $\mathrm{CP}$ violating phase determines the flavor structure of the Dirac neutrino mass matrix in the minimal seesaw model. New minimal Dirac neutrino mass matrices are presented in the case of $\mathrm{TM}_{1}$, which is given by the additional 2-3 family mixing to the tri-bimaximal mixing basis in the normal hierarchy of neutrino masses. Our model includes the Littlest seesaw model by King et al. as one of the specific cases. Furthermore, it is remarked that our $3 \times 2$ Dirac neutrino mass matrix is reproduced by introducing gauge singlet flavons with the specific alignments of the VEV's. These alignments are derived from the residual symmetry of $S_{4}$ group.

Keywords: Neutrino Physics, CP violation, Discrete Symmetries

ARXIV EPRINT: 1709.02136 


\section{Contents}

1 Introduction 1

2 Realization of minimal seesaw model 3

$2.1 \mathrm{TM}_{1}$ : 2-3 family mixing in $\mathrm{NH}$

$2.2 \mathrm{TM}_{1}$ : 2-3 family mixing in $\mathrm{IH} \quad 6$

$2.3 \mathrm{TM}_{2}$ : 1-3 family mixing in $\mathrm{NH}$ or $\mathrm{IH} \quad 7$

$\begin{array}{lll}2.4 & \text { Dirac neutrino mass matrix and vacuum alignment of flavons } & 7\end{array}$

3 Numerical results $\quad 9$

3.1 Three cases of $\mathrm{TM}_{1}$ (2-3 family mixing) in NH 10

3.2 General case for $\mathrm{TM}_{1}$ (2-3 family mixing) in $\mathrm{NH} \quad 14$

$3.3 \mathrm{TM}_{1}$ : 2-3 family mixing in $\mathrm{IH} \quad 15$

$3.4 \mathrm{TM}_{2}$ : 1-3 family mixing in $\mathrm{NH}$ or $\mathrm{IH} \quad 16$

$\begin{array}{lll}4 & \text { Summary and discussions } & 17\end{array}$

A Minimal seesaw mass matrix $\quad 19$

$\begin{array}{lll}\text { A.1 } \mathrm{TM}_{1} \text { : additional 2-3 family rotation in } \mathrm{NH} & 19\end{array}$

A.2 $\mathrm{TM}_{1}$ : additional 2-3 rotation in IH 21

A.3 $\mathrm{TM}_{2}$ : additional 1-3 rotation in $\mathrm{NH}$ or $\mathrm{IH} \quad 21$

B Representations of $S_{4}$ group $\quad 22$

\section{Introduction}

The standard model (SM) has been well established by the discovery of the Higgs boson. However, the origin of flavor in quarks and leptons is still unknown in spite of the remarkable success of the SM. The underlying physics for the flavor in quarks and leptons is one of the fundamental problems in particle physics.

On the other hand, the neutrino oscillation experiments are going on a new step to observe the $\mathrm{CP}$ violation in the lepton sector. The T2K experiment has confirmed the neutrino oscillation in the $\nu_{\mu} \rightarrow \nu_{e}$ appearance events [1], which provides us a new information of the $\mathrm{CP}$ violation in the lepton sector. Recent T2K data strongly indicate the $\mathrm{CP}$ violation in the neutrino oscillation $[2,3]$. The $\mathrm{NO} \nu \mathrm{A}$ data also suggest the $\mathrm{CP}$ violation [4], which is consistent with the T2K result. Since the experimental data of the $\mathrm{CP}$ violating phase will be available in the near future, one can develop the flavor structure of Yukawa couplings by taking account of it. 
We study the flavor structure in the seesaw model [5]-[8-10] in order to find a clue of the underlying physics of flavor. It is advantageous to consider the minimum number of parameters needed for reproducing the neutrino mixing angles and CP violating phases completely [11]. Indeed, there are some attempts toward so-called the minimal seesaw model [12]-[24].

In this work, we investigate the minimal seesaw model via the $\mathrm{CP}$ violation, where we assume two right-handed Majorana neutrinos. In addition, we take the trimaximal mixing pattern $[25,26]$ of the neutrino flavor to reduce the number of free parameters of the Dirac neutrino mass matrix. The trimaximal mixing pattern is derived from the flavor symmetry.

Before the reactor experiments reported the non-zero value of $\theta_{13}$ in 2012 [27, 28], there has been a paradigm of the tri-bimaximal (TBM) mixing [29, 30]. Since the TBM mixing pattern is a highly symmetric, the non-Abelian discrete groups have become the center of attention at the flavor symmetry [31]-[38]. The observation of the non-vanishing $\theta_{13}$ forces to study the deviation from the TBM mixing. It is remarked that the trimaximal mixing pattern is still available after the observation of $\theta_{13}$. The trimaximal mixing is given by the additional rotation of 2-3 $\left(\mathrm{TM}_{1}\right)$ or 1-3 $\left(\mathrm{TM}_{2}\right)$ families of neutrinos to the TBM mixing basis $[39,40]$. The additional rotation of 1-2 families is called as $\mathrm{TM}_{3}$, however it still leads to $\theta_{13}=0$. We do not discuss the additional rotation of 1-2 families, $\mathrm{TM}_{3}$, since it leads to $\theta_{13}=0$.

One obtains testable relations among the neutrino mixing angles and the $\mathrm{CP}$ violating phase, so-called mixing sum rules in $\mathrm{TM}_{1}$ and $\mathrm{TM}_{2}$ [41]-[43]. The prediction for the $\mathrm{CP}$ violation of neutrinos has a big impact on the study of the flavor structure [44]-[55] because $\mathrm{T} 2 \mathrm{~K}$ and $\mathrm{NO} \nu \mathrm{A}$ experiments are expected to confirm the non-zero $\mathrm{CP}$ violating phase in the near future. We discuss the $\mathrm{CP}$ violation of neutrinos for both $\mathrm{TM}_{1}$ and $\mathrm{TM}_{2}$ in the framework of the minimal seesaw model to restrict the structure of the Dirac neutrino mass matrix. It is found that those Dirac neutrino mass matrices are reproduced by introducing gauge singlet flavons with the vacuum expectation value (VEV) in the non-Abelian discrete symmetry, for example, $S_{4}$ symmetry. The specific alignments of the VEV's suggest the residual symmetry of $S_{4}$ group in our work.

We investigate the Dirac neutrino mass matrix in the diagonal basis of the charged lepton mass matrix and the $2 \times 2$ right-handed Majorana neutrino mass matrix focusing the CP violating Dirac phase. Since our results depend on the neutrino mass hierarchies, we discuss both cases of the normal hierarchy $(\mathrm{NH})$ and the inverted hierarchy (IH) for neutrino masses, respectively.

Our analyses include the Littlest seesaw model by King et al. [56]-[58] as the one of the specific cases of our model.

The paper is organized as follows. We present our framework of the minimal seesaw model in section 2, where the structure of the Dirac neutrino mass matrix is discussed to reproduce $\mathrm{TM}_{1}$ and $\mathrm{TM}_{2}$ for both cases of $\mathrm{NH}$ and $\mathrm{IH}$. The Dirac neutrino mass matrix is also discussed in the view of the flavor symmetry. In section 3, the numerical results are presented. The section 4 is devoted to the summary and discussions. Appendix A gives the detail studies of the Dirac neutrino mass matrix, and appendix B presents the necessary group theory of $S_{4}$. 


\section{Realization of minimal seesaw model}

Let us start with discussing the structure of the lepton mixing matrix, so-called the Pontecorvo-Maki-Nakagawa-Sakata (PMNS) matrix $U_{\mathrm{PMNS}}[59,60]$, where neutrinos are supposed to be Majorana particles. It is parametrized in terms of three mixing angles $\theta_{i j}$ $(i, j=1,2,3 ; i<j)$, one $\mathrm{CP}$ violating Dirac phase $\delta_{\mathrm{CP}}$, and two Majorana phases $\alpha, \beta$ as follows:

$$
U_{\mathrm{PMNS}} \equiv\left(\begin{array}{ccc}
c_{12} c_{13} & s_{12} c_{13} & s_{13} e^{-i \delta_{\mathrm{CP}}} \\
-s_{12} c_{23}-c_{12} s_{23} s_{13} e^{i \delta_{\mathrm{CP}}} & c_{12} c_{23}-s_{12} s_{23} s_{13} e^{i \delta_{\mathrm{CP}}} & s_{23} c_{13} \\
s_{12} s_{23}-c_{12} c_{23} s_{13} e^{i \delta_{\mathrm{CP}}} & -c_{12} s_{23}-s_{12} c_{23} s_{13} e^{i \delta_{\mathrm{CP}}} & c_{23} c_{13}
\end{array}\right) \times\left(\begin{array}{ccc}
e^{i \frac{\alpha}{2}} & 0 & 0 \\
0 & e^{i \frac{\beta}{2}} & 0 \\
0 & 0 & 1
\end{array}\right),
$$

where $c_{i j}$ and $s_{i j}$ denote $\cos \theta_{i j}$ and $\sin \theta_{i j}$, respectively. The CP violating measure, Jarlskog invariant [61], is defined by the PMNS matrix elements $U_{\alpha i}$, and is written in terms of the mixing angles and the $\mathrm{CP}$ violating phase as:

$$
J_{\mathrm{CP}}=\operatorname{Im}\left[U_{e 1} U_{\mu 2} U_{e 2}^{*} U_{\mu 1}^{*}\right]=s_{23} c_{23} s_{12} c_{12} s_{13} c_{13}^{2} \sin \delta_{\mathrm{CP}} .
$$

For the lepton mixing matrix, Harrison-Perkins-Scott proposed a simple form of the mixing matrix, so-called TBM mixing $[29,30]$ as follows:

$$
V_{\mathrm{TBM}}=\left(\begin{array}{ccc}
\frac{2}{\sqrt{6}} & \frac{1}{\sqrt{3}} & 0 \\
-\frac{1}{\sqrt{6}} & \frac{1}{\sqrt{3}} & -\frac{1}{\sqrt{2}} \\
-\frac{1}{\sqrt{6}} & \frac{1}{\sqrt{3}} & \frac{1}{\sqrt{2}}
\end{array}\right)
$$

which was a good scheme for the lepton sector before the reactor experiments reported non-zero $\theta_{13}$. Therefore, it is reasonable to take the TBM mixing as the starting point of the lepton mixing. In order to avoid vanishing $\theta_{13}$, we move to the trimaximal mixing basis from the TBM mixing basis.

The minimal seesaw model consists of two right-handed Majorana neutrinos and three left-handed neutrinos in Type I seesaw [11]. Taking both the charged lepton mass matrix and right-handed Majorana neutrino one $M_{R}$ to be real diagonal, $M_{R}$ and the Dirac neutrino mass matrix $M_{D}$ are generally written as:

$$
M_{R}=-M_{0}\left(\begin{array}{cc}
p^{-1} & 0 \\
0 & 1
\end{array}\right), \quad M_{D}=\left(\begin{array}{ll}
a & d \\
b & e \\
c & f
\end{array}\right),
$$

respectively, where $a \sim f$ are complex parameters and $p$ is the ratio between the two righthanded Majorana neutrino masses. The minus sign in front of $M_{0}$ is taken as our sign convention. By using the seesaw mechanism of Type I, the left-handed Majorana neutrino mass matrix $M_{\nu}$ is give by

$$
M_{\nu}=-M_{D} M_{R}^{-1} M_{D}^{T}=\frac{1}{M_{0}}\left(\begin{array}{ccc}
a^{2} p+d^{2} & a b p+d e & a c p+d f \\
a b p+d e & b^{2} p+e^{2} & b c p+e f \\
a c p+d f & b c p+e f & c^{2} p+f^{2}
\end{array}\right)
$$


By turning the neutrino mass matrix $M_{\nu}$ to the TBM mixing basis, the left-handed Majorana neutrino mass matrix is given as

$$
\hat{M}_{\nu} \equiv V_{\mathrm{TBM}}^{T} M_{\nu} V_{\mathrm{TBM}}=\frac{1}{M_{0}}\left(\begin{array}{ccc}
\frac{A_{\nu}^{2} p+D_{\nu}^{2}}{6} & \frac{A_{\nu} B_{\nu} p+D_{\nu} E_{\nu}}{3 \sqrt{2}} & \frac{A_{\nu} C_{\nu} p+D_{\nu} F_{\nu}}{2 \sqrt{3}} \\
\frac{A_{\nu} B_{\nu} p+D_{\nu} E_{\nu}}{3 \sqrt{2}} & \frac{B_{\nu}^{2} p+E_{\nu}^{2}}{3} & \frac{B_{\nu} C_{\nu} p+E_{\nu} F_{\nu}}{\sqrt{6}} \\
\frac{A_{\nu} C_{\nu} p+D_{\nu} F_{\nu}}{2 \sqrt{3}} & \frac{B_{\nu} C_{\nu} p+E_{\nu} F_{\nu}}{\sqrt{6}} & \frac{C_{\nu}^{2} p+F_{\nu}^{2}}{2}
\end{array}\right)
$$

where

$$
\begin{aligned}
A_{\nu} \equiv 2 a-b-c, & B_{\nu} \equiv a+b+c, & C_{\nu} \equiv c-b, \\
D_{\nu} \equiv 2 d-e-f, & E_{\nu} \equiv d+e+f, & F_{\nu} \equiv f-e .
\end{aligned}
$$

The mass matrix $\hat{M}_{\nu}$ is the left-handed Majorana neutrino mass matrix in the TBM mixing basis. In appendix A, we have classified this neutrino mass matrix to reproduce the trimaximal mixing $\mathrm{TM}_{1}$ and $\mathrm{TM}_{2}$ by the additional rotation of 2-3 or 1-3 families for both cases of $\mathrm{NH}$ and $\mathrm{IH}$.

\section{$2.1 \quad \mathrm{TM}_{1}: 2-3$ family mixing in $\mathrm{NH}$}

First of all, we consider the case of $\mathrm{TM}_{1}$ in $\mathrm{NH}$. The non-vanishing $\theta_{13}$ is obtained by an additional 2-3 family mixing to the TBM mixing basis $[11,40]$. This case is given by the following Dirac neutrino mass matrix as shown in appendix A.1:

$$
M_{D}=\left(\begin{array}{cc}
\frac{b+c}{2} & \frac{e+f}{2} \\
b & e \\
c & f
\end{array}\right),
$$

which leads to the neutrino mass matrix

$$
\hat{M}_{\nu}=\frac{f^{2}}{M_{0}}\left(\begin{array}{lcc}
0 & 0 & 0 \\
0 & \frac{3}{4}\left[B^{2} e^{2 i \phi_{B}}(1+j)^{2}+(k+1)^{2}\right] & \frac{1}{2} \sqrt{\frac{3}{2}}\left[B^{2} e^{2 i \phi_{B}}\left(1-j^{2}\right)-k^{2}+1\right] \\
0 & \frac{1}{2} \sqrt{\frac{3}{2}}\left[B^{2} e^{2 i \phi_{B}}\left(1-j^{2}\right)-k^{2}+1\right] & \frac{1}{2}\left[B^{2} e^{2 i \phi_{B}}(1-j)^{2}+(k-1)^{2}\right]
\end{array}\right),
$$

where we put $p=1$ in eq. (2.6) after rescaling parameters. This matrix is derived from eq. (A.7) in appendix A, where we can take $e$ and $f$ to be real, and $b$ and $c$ to be complex in general by using the freedom of redefinitions of phases in the left-handed lepton fields. We consider the case that the relative phase between $b$ and $c$ is 0 or $\pi$ in order to reduce the number of parameters of the neutrino mass matrix. Then, the only one phase which leads to the $\mathrm{CP}$ violation of neutrinos is the relative phase between the first and the second columns of the Dirac neutrino mass matrix in eq. (2.8), that is the phase of $c / f$. Since we neglect the relative phase between $b$ and $c$, the effect of it is discussed in subsection 3.2. Parameters in eq. (2.9) are defined as

$$
\frac{e}{f}=k, \quad \frac{b}{c}=j, \quad \frac{c}{f}=B e^{i \phi_{B}},
$$

where $k, j$, and $B$ are real. 
The neutrino mass matrix in eq. (2.9) is diagonalized by the rotation of 2-3 families as

$$
V_{23}=\frac{1}{\mathcal{A}}\left(\begin{array}{ccc}
\mathcal{A} & 0 & 0 \\
0 & 1 & \mathcal{V} \\
0 & -\mathcal{V}^{*} & 1
\end{array}\right), \quad \mathcal{A}=\sqrt{1+|\mathcal{V}|^{2}}
$$

where $\mathcal{V}$ is given in terms of $k, j, B$, and $\phi_{B}$. Therefore, the PMNS matrix is calculated as follows:

$$
U_{\mathrm{PMNS}}=V_{\mathrm{TBM}} V_{23}
$$

which gives three mixing angles, one Dirac phase, and one Majorana phase.

On the other hand, three neutrino masses are related to those parameters as follows:

$$
\begin{aligned}
m_{1} & =0, \quad m_{2}^{2} m_{3}^{2}=\frac{9}{4}(j-k)^{4} B^{4} f^{8}, \\
m_{2}^{2}+m_{3}^{2} & =\frac{f^{4}}{16}\left[B^{4}\left(5 j^{2}+2 j+5\right)^{2}+2 B^{2}(5 j k+j+k+5)^{2} \cos 2 \phi_{B}+\left(5 k^{2}+2 k+5\right)^{2}\right],
\end{aligned}
$$

which indicate that the PMNS matrix elements are correlated with neutrino masses.

At first, we study the specific Dirac neutrino mass matrices where the relative phase of $b / c$ vanishes. These cases lead to one zero textures for the Dirac neutrino mass matrix (see appendix A). For case I, we consider the case of $j=b / c=-1$. The neutrino mass matrix is given as

$$
\text { Case I : } \hat{M}_{\nu}=\frac{f^{2}}{M_{0}}\left(\begin{array}{ccc}
0 & 0 & 0 \\
0 & \frac{3}{4}(k+1)^{2} & -\frac{1}{2} \sqrt{\frac{3}{2}}\left(k^{2}-1\right) \\
0 & -\frac{1}{2} \sqrt{\frac{3}{2}}\left(k^{2}-1\right) & 2 B^{2} e^{2 i \phi_{B}}+\frac{1}{2}(k-1)^{2}
\end{array}\right)
$$

where the Dirac neutrino mass matrix is

$$
M_{D}=\left(\begin{array}{cc}
0 & \frac{e+f}{2} \\
b & e \\
-b & f
\end{array}\right)
$$

For case II where $c=0$ is put, it is

$$
\text { Case II : } \hat{M}_{\nu}=\frac{f^{2}}{M_{0}}\left(\begin{array}{ccc}
0 & 0 & 0 \\
0 & \frac{3}{4}\left[\hat{B}^{2} e^{2 i \phi_{B}}+(k+1)^{2}\right] & -\frac{1}{2} \sqrt{\frac{3}{2}}\left[\hat{B}^{2} e^{2 i \phi_{B}}+k^{2}-1\right] \\
0 & -\frac{1}{2} \sqrt{\frac{3}{2}}\left[\hat{B}^{2} e^{2 i \phi_{B}}+k^{2}-1\right] & \frac{1}{2}\left[\hat{B}^{2} e^{2 i \phi_{B}}+(k-1)^{2}\right]
\end{array}\right),
$$

which corresponds to $j=\infty$ and $B=0$ with a finite quantity $\hat{B} \equiv B j$, that is

$$
M_{D}=\left(\begin{array}{cc}
\frac{b}{2} & \frac{e+f}{2} \\
b & e \\
0 & f
\end{array}\right)
$$


For case III where $b=0$, it is

Case III : $\hat{M}_{\nu}=\frac{f^{2}}{M_{0}}\left(\begin{array}{ccc}0 & 0 & 0 \\ 0 & \frac{3}{4}\left[B^{2} e^{2 i \phi_{B}}+(k+1)^{2}\right] & -\frac{1}{2} \sqrt{\frac{3}{2}}\left[-B^{2} e^{2 i \phi_{B}}+k^{2}-1\right] \\ 0 & -\frac{1}{2} \sqrt{\frac{3}{2}}\left[-B^{2} e^{2 i \phi_{B}}+k^{2}-1\right] & \frac{1}{2}\left[B^{2} e^{2 i \phi_{B}}+(k-1)^{2}\right]\end{array}\right)$,

which corresponds to $j=0$, that is

$$
M_{D}=\left(\begin{array}{cc}
\frac{c}{2} & \frac{e+f}{2} \\
0 & e \\
c & f
\end{array}\right) .
$$

It is interesting to note the relation between our neutrino mass matrices and the Littlest seesaw model by King et al. [56]-[58]. It corresponds to $k=-3$ in case I, that is,

$$
M_{D}=\left(\begin{array}{cc}
0 & f \\
b & 3 f \\
-b & -f
\end{array}\right) .
$$

We discuss the phenomenological implication for this model.

\section{$2.2 \quad \mathrm{TM}_{1}: 2-3$ family mixing in $\mathrm{IH}$}

Next, we discuss the IH case of neutrino masses in $\mathrm{TM}_{1}$. As shown in appendix A.2, the Dirac and left-handed Majorana neutrino mass matrices are given as:

$$
M_{D}=\left(\begin{array}{cc}
-2 b & \frac{e+f}{2} \\
b & e \\
b & f
\end{array}\right),
$$

and

$$
\hat{M}_{\nu}=\frac{1}{M_{0}}\left(\begin{array}{ccc}
6 b^{2} & 0 & 0 \\
0 & \frac{3}{4}(e+f)^{2} & -\frac{1}{2} \sqrt{\frac{3}{2}}(e-f)(e+f) \\
0 & -\frac{1}{2} \sqrt{\frac{3}{2}}(e-f)(e+f) & \frac{1}{2}(e-f)^{2}
\end{array}\right),
$$

where $m_{1}=6 b^{2} / M_{0}$ and $m_{3}=0$. By taking the elements in the first column of the Dirac neutrino mass matrix to be real, parameters $e$ and $f$ can be complex in contrast to the case of subsection 2.1. When we redefine the complex parameters $e$ and $f$ in terms of two real parameters $k$ and $\phi_{k}$ as

$$
\frac{e}{f}=k e^{i \phi_{k}},
$$

the neutrino mass matrix turns to

$$
\hat{M}_{\nu}=\frac{6 b^{2}}{M_{0}}\left(\begin{array}{lll}
1 & 0 & 0 \\
0 & 0 & 0 \\
0 & 0 & 0
\end{array}\right)+\frac{f^{2}}{M_{0}}\left(\begin{array}{ccc}
0 & 0 & 0 \\
0 & \frac{3}{4}\left(k e^{i \phi_{k}}+1\right)^{2} & -\frac{1}{2} \sqrt{\frac{3}{2}}\left(k^{2} e^{2 i \phi_{k}}-1\right) \\
0 & -\frac{1}{2} \sqrt{\frac{3}{2}}\left(k^{2} e^{2 i \phi_{k}}-1\right) & \frac{1}{2}\left(k e^{i \phi_{k}}-1\right)^{2}
\end{array}\right) .
$$


Note that the 2-3 family mixing is determined only by $k$ and $\phi_{k}$. If the relative phase between $e$ and $f$, that is $\phi_{k}$, is zero, the CP symmetry is conserved. It is remarked that the 2-3 family mixing is independent of neutrino masses $m_{1}$ and $m_{2}$ because the neutrino masses $m_{1}$ and $m_{2}$ are given in terms of $b$ and $f$, respectively. This situation makes our numerical analysis simple for the case of IH.

Besides our neutrino mass matrix, the two-zero texture for the Dirac neutrino mass matrix has been discussed in the context of the minimal seesaw model [15, 20, 21, 23]. Especially, for the IH case, it is completely consistent with the experimental data of mixing angles and masses [20]. The prediction of the CP violating phase will be discussed comparing with our result in section 3 .

\section{$2.3 \quad \mathrm{TM}_{2}$ : 1-3 family mixing in $\mathrm{NH}$ or $\mathrm{IH}$}

Let us consider the other case, in which the additional rotation of 1-3 families diagonalizes the neutrino mass matrix $\hat{M}_{\nu}$. As seen in appendix A.3, we obtain the Dirac neutrino mass matrix and the left-handed Majorana neutrino mass matrix as follows:

$$
\begin{aligned}
M_{D} & =\left(\begin{array}{ll}
b-e-f \\
b & e \\
b & f
\end{array}\right), \\
\hat{M}_{\nu} & =\frac{1}{M_{0}}\left(\begin{array}{ccc}
\frac{3}{2}(e+f)^{2} & 0 & \frac{\sqrt{3}}{2}\left(e^{2}-f^{2}\right) \\
0 & 3 b^{2} & 0 \\
\frac{\sqrt{3}}{2}\left(e^{2}-f^{2}\right) & 0 & \frac{1}{2}(e-f)^{2}
\end{array}\right),
\end{aligned}
$$

respectively. By using the same notation in eq. (2.23), $\hat{M}_{\nu}$ is rewritten as:

$$
\hat{M}_{\nu}=\frac{3 b^{2}}{M_{0}}\left(\begin{array}{lll}
0 & 0 & 0 \\
0 & 1 & 0 \\
0 & 0 & 0
\end{array}\right)+\frac{f^{2}}{M_{0}}\left(\begin{array}{ccc}
\frac{3}{2}\left(k e^{i \phi_{k}}+1\right)^{2} & 0 & \frac{\sqrt{3}}{2}\left(k^{2} e^{2 i \phi_{k}}-1\right) \\
0 & 0 & 0 \\
\frac{\sqrt{3}}{2}\left(k^{2} e^{2 i \phi_{k}}-1\right) & 0 & \frac{1}{2}\left(k e^{i \phi_{k}}-1\right)^{2}
\end{array}\right) .
$$

As well as the case of the 2-3 family mixing for IH in subsection 2.2, the 1-3 family mixing is also determined only by $k$ and $\phi_{k}$, namely, it is independent of neutrino masses.

\subsection{Dirac neutrino mass matrix and vacuum alignment of flavons}

Above specific structures of the $3 \times 2$ Dirac neutrino mass matrix are given by introducing flavons with VEV's in the framework of the non-Abelian discrete symmetry $S_{4}$. The Dirac neutrino mass matrices in subsections $2.1,2.2$, and 2.3 can be reproduced by the four types of flavons $\phi_{i}$. These four flavons are represented as triplets of $S_{4}$ group and have specific alignments of VEV's:

$$
\left\langle\phi_{i}\right\rangle=\left(\begin{array}{c}
\frac{b+c}{2} \\
c \\
b
\end{array}\right), \quad\left(\begin{array}{c}
-2 \\
1 \\
1
\end{array}\right),\left(\begin{array}{l}
1 \\
1 \\
1
\end{array}\right), \quad\left(\begin{array}{c}
-e-f \\
f \\
e
\end{array}\right)
$$


The first two VEV's in eq. (2.28) preserve the $S U(U S)$ symmetry for $\mathbf{3}^{\prime}$ and $\mathbf{3}$, respectively since the generator $S U(U S)$ is expressed as

$$
S U=U S=\mp \frac{1}{3}\left(\begin{array}{ccc}
-1 & 2 & 2 \\
2 & 2 & -1 \\
2 & -1 & 2
\end{array}\right)
$$

for $\mathbf{3}$ and $\mathbf{3}^{\prime}$, respectively. These VEV's are broken by $S, T$, and $U$ (see appendix B). Those flavons have the $Z_{2}$ symmetry with elements $\{1, S U\}$. This $Z_{2}$ symmetry is a residual one after $S_{4}$ is broken. The third VEV in eq. (2.28) holds the $S$ symmetry for both $\mathbf{3}$ and $\mathbf{3}^{\prime}$, but broken by $T$. The last VEV in eq. (2.28) is not preserved by $S, T$, nor $U$ unless $e=f$.

Let us reproduce the Dirac neutrino mass matrix in eq. (2.8) by introducing those flavons. Suppose that the left-handed lepton $L$ and the flavons $\phi_{\text {atm }}, \phi_{\text {sol }}$ are $\mathbf{3}^{\prime}$ of $S_{4}$, while the right-handed Majorana neutrinos $\nu_{R 1}$ and $\nu_{R 2}$ are $\mathbf{1}$ of $S_{4}$. The Higgs field $H_{u}$ is also 1 . For the 2-3 family mixing in NH, the Dirac neutrino mass matrix is reproduced by the Yukawa couplings

$$
\frac{y_{\mathrm{atm}}}{\Lambda} \phi_{\mathrm{atm}} L H_{u} \nu_{R 1}^{c}+\frac{y_{\mathrm{sol}}}{\Lambda} \phi_{\mathrm{sol}} L H_{u} \nu_{R 2}^{c},
$$

where $y_{\mathrm{atm}}$ and $y_{\mathrm{sol}}$ are arbitrary coupling constants, $\Lambda$ is the cut-off scale of $S_{4}$ symmetry. The VEV's of $\phi_{\text {atm }}$ and $\phi_{\text {sol }}$ are

$$
\left\langle\phi_{\mathrm{atm}}\right\rangle \sim\left(\begin{array}{c}
\frac{b+c}{2} \\
c \\
b
\end{array}\right), \quad\left\langle\phi_{\mathrm{sol}}\right\rangle \sim\left(\begin{array}{c}
\frac{e+f}{2} \\
f \\
e
\end{array}\right),
$$

since $S_{4}$ singlet contraction $\mathbf{3}^{\prime} \otimes \mathbf{3}^{\prime}$ implies that $L\left(\mathbf{3}^{\prime}\right) \phi\left(\mathbf{3}^{\prime}\right)=L_{1} \phi_{1}+L_{2} \phi_{3}+L_{3} \phi_{2}$ as seen in appendix B.

For the case of IH of $\mathrm{TM}_{1}$, the assignments of the irreducible representations should be changed from the one of $\mathrm{NH}$. We suppose that $L$ and $\phi_{\text {atm }}$ are $\mathbf{3}$, and $\phi_{\text {sol }}$ is $\mathbf{3}^{\prime}$ of $S_{4}$, while $\nu_{R 1}$ is $\mathbf{1}$ and $\nu_{R 2}$ is $\mathbf{1}^{\prime}$ of $S_{4}$. It is easily seen that the Dirac neutrino mass matrix is reproduced by the Yukawa couplings in eq. (2.30), where the VEV's of $\phi_{\text {atm }}$ and $\phi_{\text {sol }}$ are

$$
\left\langle\phi_{\mathrm{atm}}\right\rangle \sim\left(\begin{array}{c}
-2 \\
1 \\
1
\end{array}\right), \quad\left\langle\phi_{\mathrm{sol}}\right\rangle \sim\left(\begin{array}{c}
\frac{e+f}{2} \\
f \\
e
\end{array}\right)
$$

since $\mathbf{3} \otimes \mathbf{3}^{\left({ }^{\prime}\right)}$ also implies that $L(\mathbf{3}) \phi\left(\mathbf{3}^{\left({ }^{\prime}\right)}\right)=L_{1} \phi_{1}+L_{2} \phi_{3}+L_{3} \phi_{2}$ for $\mathbf{1}^{\left({ }^{\prime}\right)}$. Thus, the Dirac neutrino mass matrix is reproduced by the relevant assignments of the flavons. The $2 \times 2$ diagonal right-handed Majorana neutrino mass matrix is reproduced by help of the auxiliary $Z_{2}$ symmetry in NH while an auxiliary $Z_{2}$ is not necessary in IH. The diagonal charged lepton mass matrix is realized as well as the Littlest seesaw model [56] by introducing three 3 flavons, which have VEV's:

$$
\left\langle\phi_{e}\right\rangle \sim\left(\begin{array}{l}
1 \\
0 \\
0
\end{array}\right), \quad\left\langle\phi_{\mu}\right\rangle \sim\left(\begin{array}{l}
0 \\
1 \\
0
\end{array}\right), \quad\left\langle\phi_{\tau}\right\rangle \sim\left(\begin{array}{l}
0 \\
0 \\
1
\end{array}\right) .
$$


For $\mathrm{TM}_{2}$, that is the case of the 1-3 family mixing, the Dirac neutrino mass matrix can be reproduced by the relevant Yukawa couplings in the similar way. However, the situation of symmetry is different from the case of $\mathrm{TM}_{1}$ because the fourth VEV in eq. (2.28) is not preserved in any subgroups of $S_{4}$. In order to obtain desirable Yukawa couplings, we need an auxiliary $Z_{2}$ symmetry.

\section{Numerical results}

In this section, we discuss the numerical results for the $\mathrm{CP}$ violating Dirac phase $\delta_{\mathrm{CP}}$ as well as neutrino mixing angles. We also discuss the Majorana phases, which contribute the effective mass for the neutrinoless double beta decay $(0 \nu \beta \beta$ decay). The neutrino mixing angles are obtained in terms of the PMNS matrix elements $U_{\alpha i}$ of eq. (2.1) as follows:

$$
s_{12}^{2} \equiv \sin ^{2} \theta_{12}=\frac{\left|U_{e 2}\right|^{2}}{1-\left|U_{e 3}\right|^{2}}, \quad s_{23}^{2} \equiv \sin ^{2} \theta_{23}=\frac{\left|U_{\mu 3}\right|^{2}}{1-\left|U_{e 3}\right|^{2}}, \quad s_{13}^{2} \equiv \sin ^{2} \theta_{13}=\left|U_{e 3}\right|^{2} .
$$

The Dirac CP violating phase $\delta_{\mathrm{CP}}$ can be calculated by using the Jarlskog invariant in eq. (2.2):

$$
\sin \delta_{\mathrm{CP}}=\frac{J_{\mathrm{CP}}}{s_{23} c_{23} s_{12} c_{12} s_{13} c_{13}^{2}},
$$

where $\cos \delta_{\mathrm{CP}}$ is fixed by $\left|U_{\mu 1}\right|^{2}=1 / 6$ for the case of $2-3$ family mixing, and $\left|U_{\mu 2}\right|^{2}=1 / 3$ for the case of 1-3 family mixing, respectively.

The effective mass for the $0 \nu \beta \beta$ decay is given in terms of the Dirac phase $\delta_{\mathrm{CP}}$ and Majorana phases $\alpha$ and $\beta$ as follows:

$$
\begin{aligned}
\left|m_{e e}\right| & =\left|m_{1} U_{e 1}^{2}+m_{2} U_{e 2}^{2}+m_{3} U_{e 3}^{2}\right| \\
& =\left|m_{1} c_{13}^{2} c_{12}^{2} e^{i \alpha}+m_{2} c_{13}^{2} s_{12}^{2} e^{i \beta}+m_{3} s_{13}^{2} e^{-2 i \delta_{\mathrm{CP}}}\right|,
\end{aligned}
$$

where $m_{1}=0$ or $m_{3}=0$ for $\mathrm{NH}$ or $\mathrm{IH}$, respectively. The Majorana phase $\beta$ is directly related to the expression of the effective mass $\left|m_{e e}\right|$ for $\mathrm{NH}$, while $(\alpha-\beta)$ is related to $\left|m_{e e}\right|$ for IH.

Let us explain how to obtain our predictions of the CP violation taking the case I of subsection $2.1\left(\mathrm{TM}_{1}\right.$ in $\left.\mathrm{NH}\right)$ as an example. The result of global analyses is often used as the inputting data to constrain the unknown parameters [62, 63]. In our calculations, we have adopted the result in ref. [63]. At first, by inputting the data of $\Delta m_{13}^{2}$ and $\Delta m_{12}^{2}$ within $3 \sigma(1 \sigma)$ range in table 1 , we remove the two free parameters $f^{2} / M_{0}$ and $B$ in eq. (2.14). The remained free parameter $k$ is scanned in the region of $-20 \sim 20$ by generating random numbers in the linear scale. On the other hand, the phase $\phi_{B}$ is also scanned in the full region of $-\pi \sim \pi$ in the linear scale. Then, we calculate three neutrino mixing angles. These calculated values are judged by using the experimental data within $3 \sigma(1 \sigma)$ range as shown in table 1. If they are allowed for the experimental data, we keep the point, in which the CP violating phases and $\left|m_{e e}\right|$ are calculated. Otherwise, we throw the point. We continue this procedure to obtain enough points for plotting allowed region.

In the next subsections, we show the numerical results in the case of $\mathrm{TM}_{1}$ and $\mathrm{TM}_{2}$ for both $\mathrm{NH}$ and $\mathrm{IH}$. 


\begin{tabular}{|c|cc|cc|}
\hline observable & NH: $1 \sigma$ & $3 \sigma$ & IH: $1 \sigma$ & $3 \sigma$ \\
\hline$\left|\Delta m_{13}^{2}\right| \times 10^{3}\left[\mathrm{eV}^{2}\right]$ & $2.55 \pm 0.04$, & $2.43 \sim 2.67$ & $2.49 \pm 0.04$, & $2.37 \sim 2.61$ \\
\hline$\Delta m_{12}^{2} \times 10^{5}\left[\mathrm{eV}^{2}\right]$ & $7.56 \pm 0.19$, & $7.05 \sim 8.14$ & $7.56 \pm 0.19$, & $7.05 \sim 8.14$ \\
\hline $\sin ^{2} \theta_{23}$ & $0.430_{-0.018}^{+0.020}$, & $0.384 \sim 0.635$ & $0.596_{-0.018}^{+0.017}$, & $0.388 \sim 0.638$ \\
\hline $\sin ^{2} \theta_{12}$ & $0.321_{-0.016}^{+0.018}$, & $0.273 \sim 0.379$ & $0.321_{-0.016}^{+0.018}$, & $0.273 \sim 0.379$ \\
\hline $\sin ^{2} \theta_{13}\left(10^{-2}\right)$ & $2.155_{-0.075}^{+0.090}$, & $1.89 \sim 2.39$ & $2.140_{-0.085}^{+0.082}$, & $1.89 \sim 2.39$ \\
\hline
\end{tabular}

Table 1. $1 \sigma$ and $3 \sigma$ ranges of the global analysis of the neutrino oscillation experimental data for $\mathrm{NH}$ and $\mathrm{IH}$, respectively [63].

\subsection{Three cases of $\mathrm{TM}_{1}(2-3$ family mixing) in $\mathrm{NH}$}

Let us show numerical results of case I, II, and III in subsection 2.1. The most interesting case is the case I. The case I corresponds to one of the one-zero texture models, where the $(1,1)$ element of the Dirac neutrino mass matrix is zero. There are two free parameters, $k$ and $\phi_{B}$ after inputting data of neutrino masses in table 1. As seen in figure 1(a), the prediction of $\delta_{\mathrm{CP}}$ versus $\sin ^{2} \theta_{23}$ is same as the previous result of $\mathrm{TM}_{1}$ [48], where the neutrino mass matrix and the neutrino mass hierarchy have not been specified. Unless the magnitude of the dimensionless parameter $k$ is fixed, the predicted $\sin ^{2} \theta_{23}$ is allowed to take all values within the $3 \sigma$ range of the experimental data while $\delta_{\mathrm{CP}}$ is in the region of $\pm\left(45^{\circ} \sim 125^{\circ}\right)$ depending on $\sin ^{2} \theta_{23}$ for the inputting data within $3 \sigma$. It is helpful to show the predictions for the inputting data within $1 \sigma$. The predicted $\delta_{\mathrm{CP}}$ is in the region of $\pm\left(100^{\circ} \sim 115^{\circ}\right)$ for the $1 \sigma$ range of $\sin ^{2} \theta_{23}=0.412-0.450$. It is remarked that $\left|\delta_{\mathrm{CP}}\right|$ should be larger than $90^{\circ}$ if the observed $\theta_{23}$ is in the first octant. This prediction is consistent with the recent data of $\mathrm{T} 2 \mathrm{~K}$, which present the allowed $2 \sigma$ range for the $\mathrm{CP}$ violating phase, $\delta_{\mathrm{CP}}$, such as $\left(-171^{\circ},-34.4^{\circ}\right)$ for $\mathrm{NH}[3]$.

As well known, the predicted $\sin ^{2} \theta_{12}$ is $0.317 \sim 0.320$ [48], which is a characteristic one for $\mathrm{TM}_{1}$ independent of details of the neutrino mass matrix. This predicted value is inside the region of the experimental data of $1 \sigma$ range.

We also show the result for the Majorana phase $\beta$ in figure $1(\mathrm{~b})$. For the inputting data within $3 \sigma, \beta=0$ is allowed, but $\beta=0$ is excluded for the inputting data with $1 \sigma$. It is found that $\beta$ is zero when the Dirac CP violating phase $\delta_{\mathrm{CP}}$ is $\pm \pi / 2$. The remaining Majorana phase $\alpha$ is arbitrary because of $m_{1}=0$.

It is remarked that our predictions depend on the parameter $k$. As seen in figure 1(c), it is allowed in two separated region, $k \simeq-12 \sim-2$ and $k \simeq-0.1 \sim-0.5$ for the inputting data within $3 \sigma$. These regions are related to inverse values each other. The predicted value of $\sin ^{2} \theta_{23}$ crucially depends on $k$ as seen in figure $1(\mathrm{c})$. Indeed, for the inputting data within $1 \sigma, k$ is severely constrained around -2.5 and -0.4 .

We also present $k$ dependence of $\delta_{\mathrm{CP}}$ in figure $1(\mathrm{~d})$. If the observed $\left|\delta_{\mathrm{CP}}\right|$ is larger than $90^{\circ}, k$ is larger than -3 . If $\delta_{\mathrm{CP}}$ is observed accurately as well as $\sin ^{2} \theta_{23}, k$ is determined with two-fold ambiguity. The $k$ dependence is important because textures of neutrino mass matrix are given by fixing $k$ as discussing later. 
(a)

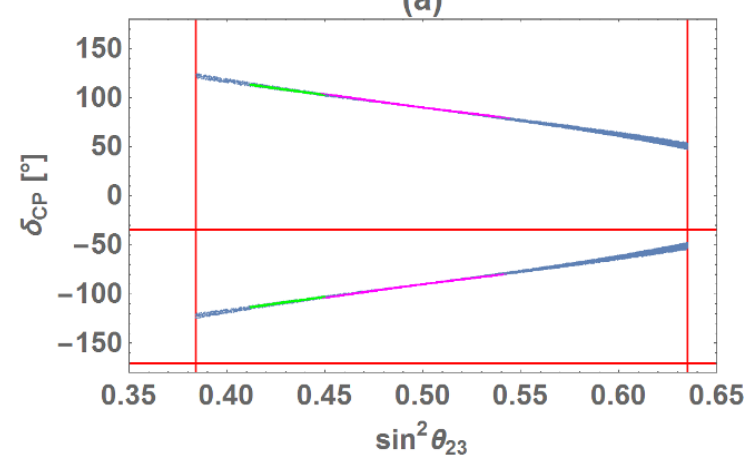

(c)

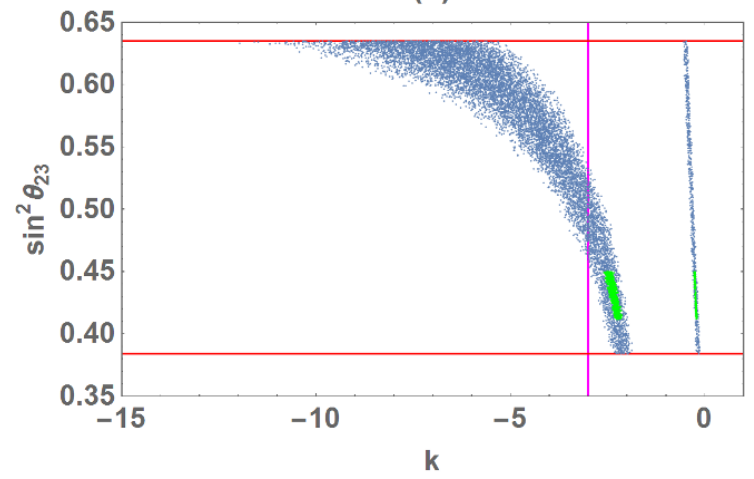

(e)

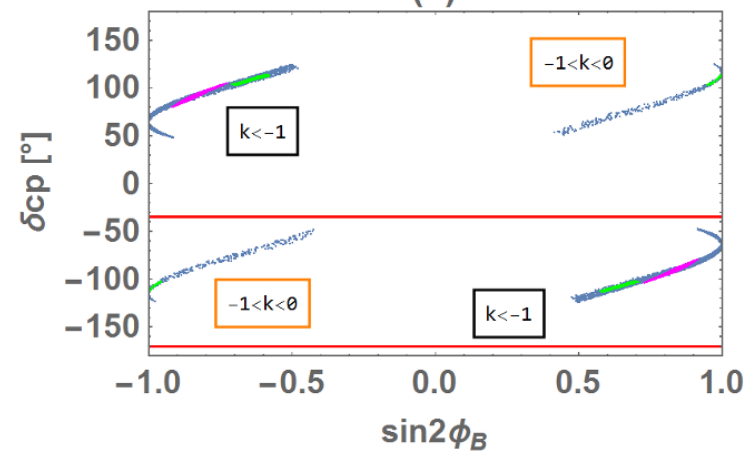

(b)

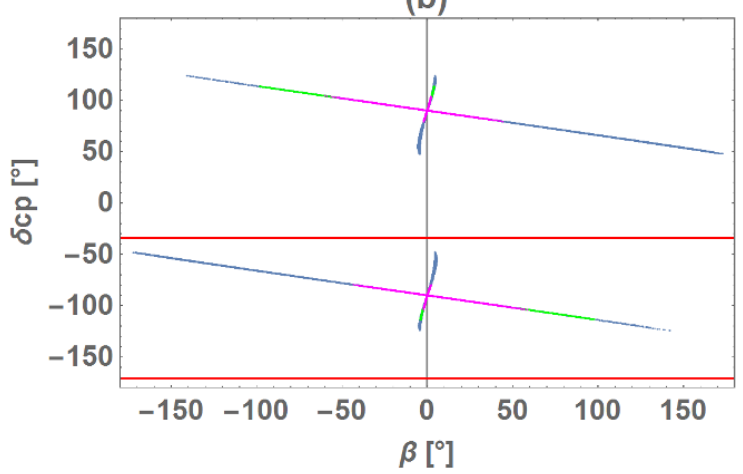

(d)

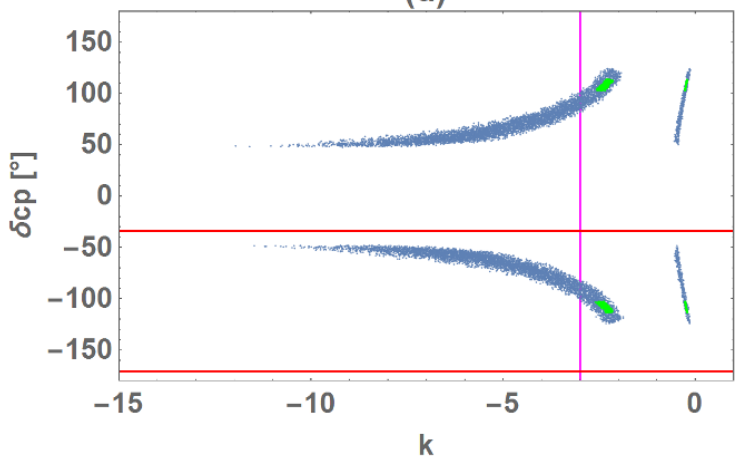

(f)

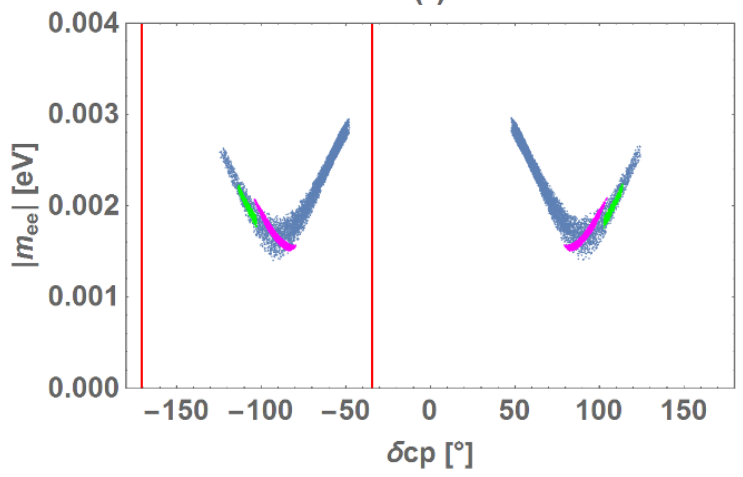

Figure 1. Blue (green) dots denote predictions for case I within $3 \sigma(1 \sigma)$ range. The red lines for $\sin ^{2} \theta_{23}$ and $\delta_{\mathrm{CP}}$ denote the experimental bounds of $3 \sigma$ (global analyses) and $2 \sigma$ (T2K) ranges, respectively: (a) $\delta_{\mathrm{CP}}$ versus $\sin ^{2} \theta_{23}$, (b) $\delta_{\mathrm{CP}}$ versus $\beta$, (c) $\sin ^{2} \theta_{23}$ versus $k$, (d) $\delta_{\mathrm{CP}}$ versus $k$, (e) $\delta_{\mathrm{CP}}$ versus $\sin 2 \phi_{B}$, and (f) $\left|m_{e e}\right|$ versus $\delta_{\mathrm{CP}}$. The magenta dots in (a), (b), (e) and (f) denote the predictions of the Littlest seesaw model. The vertical magenta lines of $k=-3$ in (c) and (d) also denote its predictions.

We present the $\sin 2 \phi_{B}$ dependence of $\delta_{\mathrm{CP}}$ in figure $1(\mathrm{e})$, where the prediction is classified by $k$. It is remarked that the sign of $\delta_{\mathrm{CP}}$ depends on both $\sin 2 \phi_{B}$ and $k$. The sign of $\delta_{\mathrm{CP}}$ is determined by the sign of $\left(\sin 2 \phi_{B}\right)$ for $k>-1$ while by the sign of $\left(-\sin 2 \phi_{B}\right)$ for $k<-1$. This result is important for model-buildings.

We show the effective mass for the $0 \nu \beta \beta$ decay $\left|m_{e e}\right|$ versus $\delta_{\mathrm{CP}}$ in figure $1(\mathrm{f})$, where $\left|m_{e e}\right|$ is around $1.5 \sim 3.0 \mathrm{meV}$. It is minimal if the $\mathrm{CP}$ violating phase $\delta_{\mathrm{CP}}$ is $\pm \pi / 2$. 
(a)

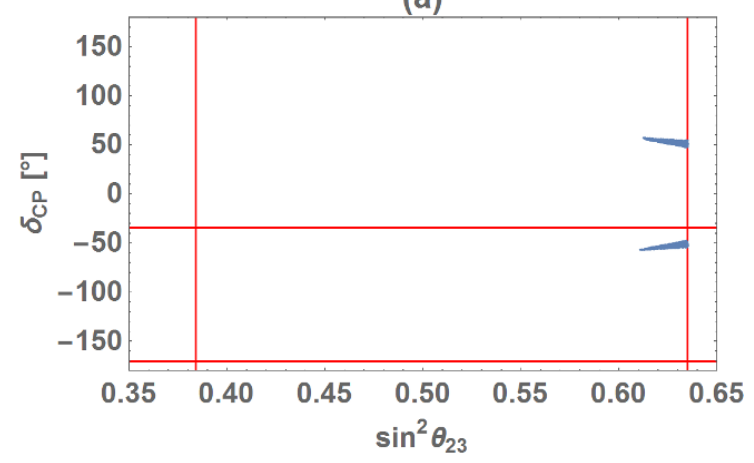

(b)

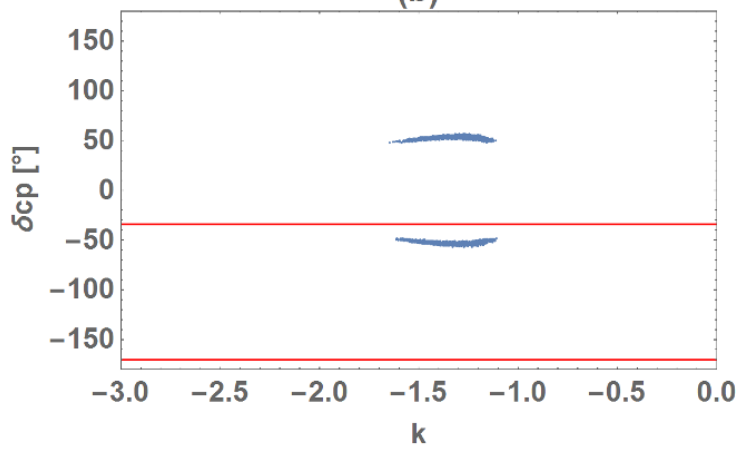

Figure 2. Predictions for case II, where the red lines for $\sin ^{2} \theta_{23}$ and $\delta_{\mathrm{CP}}$ denote the experimental bounds of $3 \sigma$ (global analyses) and $2 \sigma$ (T2K) ranges, respectively: (a) $\delta_{\mathrm{CP}}$ versus $\sin ^{2} \theta_{23}$ and (b) $\delta_{\mathrm{CP}}$ versus $k$.

The correlation between $\left|m_{e e}\right|$ and $\delta_{\mathrm{CP}}$ is helpful to test our model for the Dirac neutrino mass matrix.

Finally, we discuss the Littlest seesaw model [56]-[58] in eq. (2.20) of subsection 2.1. This corresponds to $k=-3$ in the case I. As already presented in ref. [56], this model leads to the predictions around $\sin ^{2} \theta_{23}=1 / 2$ and the maximal $\delta_{\mathrm{CP}}= \pm \pi / 2$. We show the results of the Littlest seesaw model by the color of magenta in figures 1(a)-(f). As seen in figure $1(\mathrm{a})$, the predicted $\sin ^{2} \theta_{23}$ and $\delta_{\mathrm{CP}}$ are $0.451 \sim 0.544$ and $\pm\left(80^{\circ} \sim 105^{\circ}\right)$, respectively. As seen in figure 1(b), the Majorana phase $\beta$ is in the range from $-60^{\circ}$ to $60^{\circ}$. In both figures $1(\mathrm{c})$ and $(\mathrm{d})$, the vertical magenta lines of $k=-3$ denote the predictions of the Littlest seesaw model. The effective mass $\left|m_{e e}\right|$ is in the minimal regions in figure 1(f).

We propose new Littlest seesaw models by observing the result of figure 1. For example, $k=-5$ or $-2(-0.2$ or -0.5$)$ give different predictions for $\sin ^{2} \theta_{23}$ and $\delta_{\mathrm{CP}}$. The Dirac neutrino mass matrices are

$$
M_{D}=\left(\begin{array}{cc}
0 & 2 f \\
b & 5 f \\
-b & -f
\end{array}\right), \quad\left(\begin{array}{cc}
0 & 2 f \\
b & -f \\
-b & 5 f
\end{array}\right), \quad\left(\begin{array}{cc}
0 & f \\
b & 4 f \\
-b & -2 f
\end{array}\right), \quad\left(\begin{array}{cc}
0 & f \\
b & -2 f \\
-b & 4 f
\end{array}\right)
$$

which will be testable in the future experiments. Thus, our analyses present new Dirac neutrino mass matrices, which suggest new models of the lepton flavors.

Next, we discuss the numerical results for the case II, where the $(2,1)$ element of the Dirac neutrino mass matrix is zero. In figure $2(\mathrm{a})$, the predicted $\sin ^{2} \theta_{23}$ is restricted near the upper bound of the $3 \sigma$ range of the experimental data and the $\mathrm{CP}$ violating phase is $\delta_{\mathrm{CP}} \simeq \pm 50^{\circ}$. The case II may be excluded if the more precise data of $\sin ^{2} \theta_{23}$ are available in the near future.

Although the prediction of $\delta_{\mathrm{CP}}$ in figure 2(a) seems to be in the partial regions of the one in the case I, the parameter $k$ of the case II is much different from the range of $k$ in case I, that is, $k=-1.65 \sim-1.10$ as seen in figure $2(\mathrm{~b})$. We find that the sign of $\delta_{\mathrm{CP}}$ is determined by the sign of $\left(\sin 2 \phi_{B}\right)$ in the region of $k=-1.65 \sim-1.10$. 
(a)

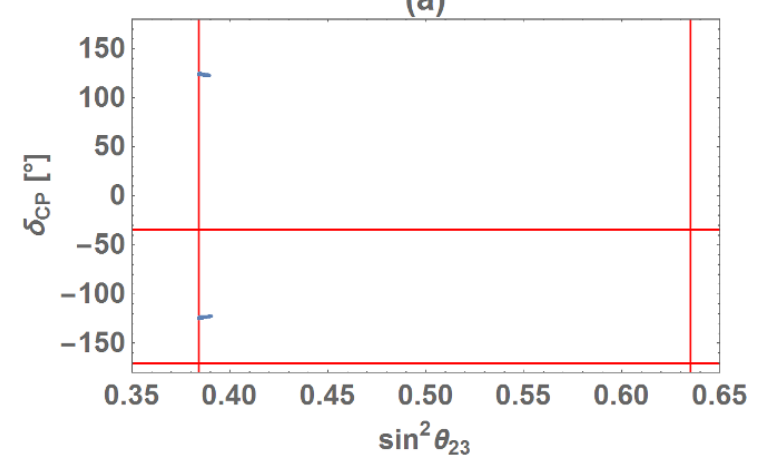

(b)

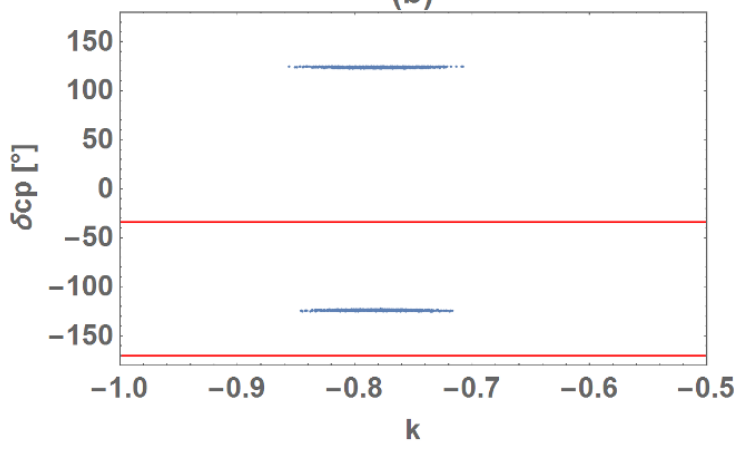

Figure 3. Predictions for case III, where the red lines for $\sin ^{2} \theta_{23}$ and $\delta_{\mathrm{CP}}$ denote the experimental bounds of $3 \sigma$ (global analyses) and $2 \sigma$ (T2K) ranges, respectively: (a) $\delta_{\mathrm{CP}}$ versus $\sin ^{2} \theta_{23}$ and (b) $\delta_{\mathrm{CP}}$ versus $k$.

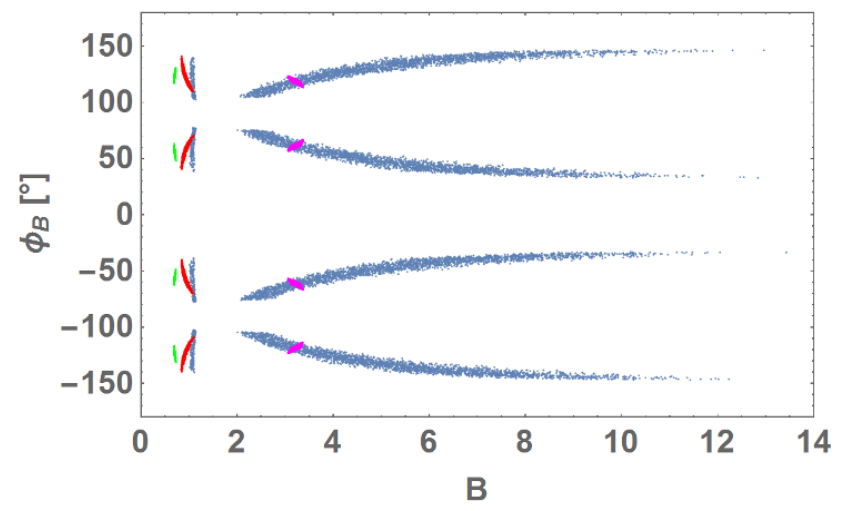

Figure 4. Allowed regions in $B-\phi_{B}$ plane for $\mathrm{TM}_{1}$ in $\mathrm{NH}$. The blue, red, green, and magenta dots denote the case I, II, III, and the Littlest seesaw model, respectively.

We note the Majorana phase and the effective mass for the $0 \nu \beta \beta$ decay. The Majorana phase $\beta$ is restricted to the region around $\pm 5^{\circ}$ and $\pm\left(140^{\circ} \sim 170^{\circ}\right)$. The effective mass $\left|m_{e e}\right|$ is predicted to be $2.6 \sim 3.0 \mathrm{meV}$.

In the case III, the $(3,1)$ element of the Dirac neutrino mass matrix is zero. In contrast to the case II, the mixing angle $\sin ^{2} \theta_{23}$ is strongly restricted to the lower bound of the $3 \sigma$ range as seen in figure 3(a). The case III is almost excluded in the present status of the neutrino oscillation experiments. We find $k=-0.86 \sim-0.71$. In this region, the Dirac $\mathrm{CP}$ violating phase $\delta_{\mathrm{CP}}$ is predicted in the region around $\pm 125^{\circ}$. The Majorana phase $\beta$ is predicted in the region around $\pm 5^{\circ}$ and $\pm 140^{\circ}$. It is also remarked that the sign of $\delta_{\mathrm{CP}}$ is determined by the sign of $\left(-\sin 2 \phi_{B}\right)$ in the region of $k=-0.86 \sim-0.71$. The predicted effective mass $\left|m_{e e}\right|$ is around $2.7 \mathrm{meV}$.

Finally in this subsection, we discuss allowed region of the parameters $B$ and $\phi_{B}$ in eq. (2.10). We show the combined results for cases I, II, III, and the Littlest seesaw model in figure 4, where the blue, red, green, and magenta dots denote the case I, II, III, and the Littlest seesaw model, respectively. Thus, the allowed regions of those parameters are clearly different each other. 
(a)

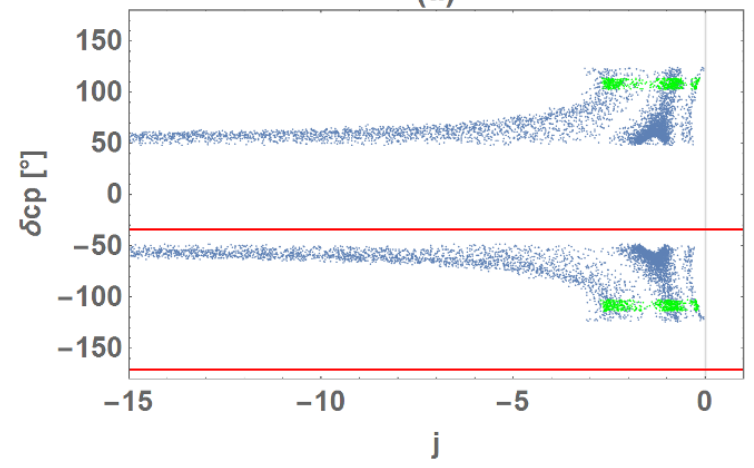

(c)

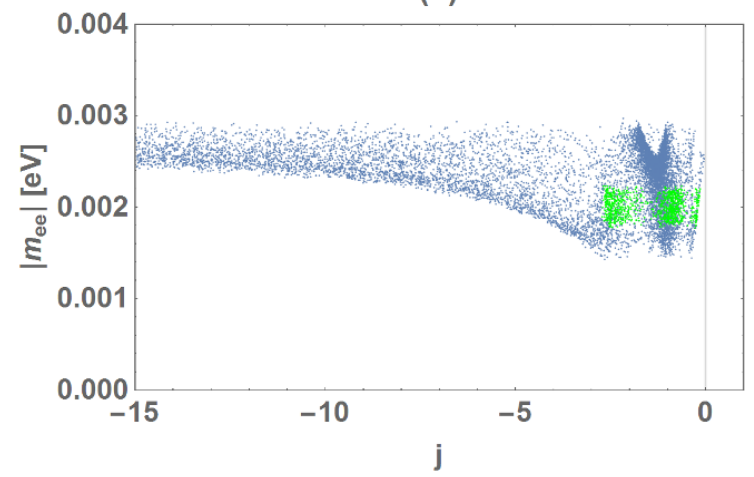

(b)

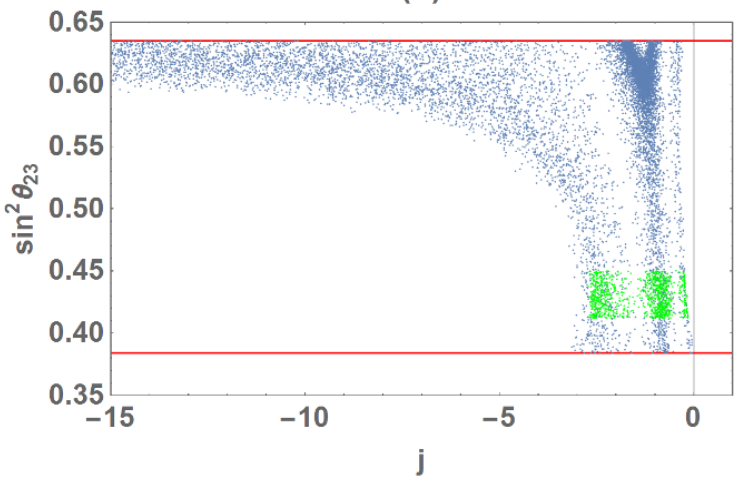

(d)

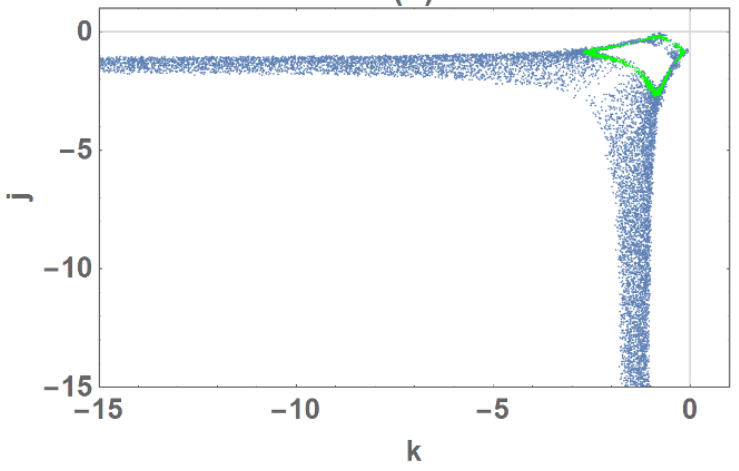

Figure 5. Blue (green) dots denote predictions for arbitrary $j$ in $\mathrm{TM}_{1}(\mathrm{NH})$ within $3 \sigma(1 \sigma)$. The red lines for $\sin ^{2} \theta_{23}$ and $\delta_{\mathrm{CP}}$ denote the experimental bounds of $3 \sigma$ (global analyses) and $2 \sigma$ (T2K) ranges, respectively: (a) $\delta_{\mathrm{CP}}$ versus $j$, (b) $\sin ^{2} \theta_{23}$ versus $j$, (c) $\left|m_{e e}\right|$ versus $j$, and (d) $k$ versus $j$.

\subsection{General case for $\mathrm{TM}_{1}(2-3$ family mixing) in $\mathrm{NH}$}

In the previous subsection, $j \equiv b / c$ has been fixed. The cases I, II, and III are specific cases for $j$, which correspond to $j=-1,-\infty$, and 0 , respectively. In order to show $j$ dependence of our predictions in detail, we discuss the case in which $j$ is not specified as in eq. (2.9).

We show the prediction of $\delta_{\mathrm{CP}}$ versus $j$ in figure $5(\mathrm{a})$, where $j$ is allowed in negative value. As $|j|$ increases, $\delta_{\mathrm{CP}}$ reaches $\pm 45^{\circ}$ asymptotically. We show the prediction of $\sin ^{2} \theta_{23}$ versus $j$ in figure $5(\mathrm{~b})$. As far as $j$ is larger than $-3, \sin ^{2} \theta_{23}$ is allowed to take all values within the $3 \sigma$ range of the experimental data. However, it is predicted to be larger than 0.5 when $j$ is smaller than -3 . It is also easily seen that the cases II $(j=-\infty)$ and III $(j=0)$ are marginal for the prediction of $\sin ^{2} \theta_{23}$ as seen in figure $5(\mathrm{~b})$. We show the prediction of $\left|m_{e e}\right|$ versus $j$ in figure 5(c). We find that the predicted region of $\left|m_{e e}\right|$ never enlarge compared with those predictions of cases I, II, and III even if $j$ is taken to be arbitrary. Finally, we show the allowed region in $k$ and $j$ plane in figure $5(\mathrm{~d})$. It is verified numerically that $j$ and $k$ are symmetric for their exchange. The allowed regions within $1 \sigma$ are rather narrow as seen in figure 5. If the error-bar of the inputting data are reduced in the future, both $k$ and $j$ are expected to be constrained considerably. 
(a)

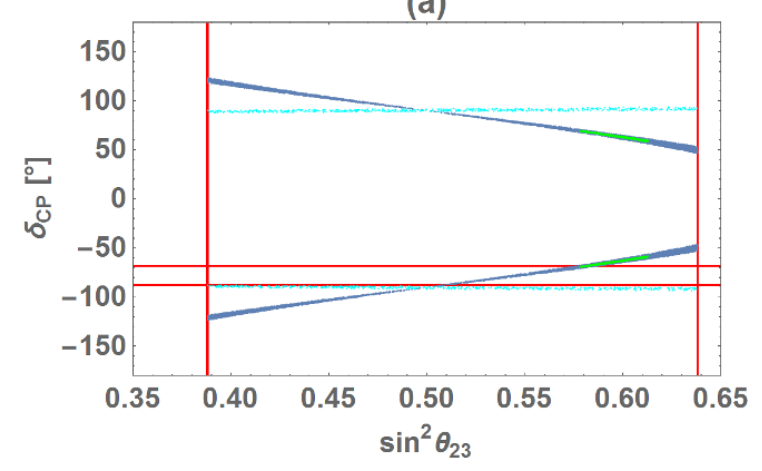

(c)

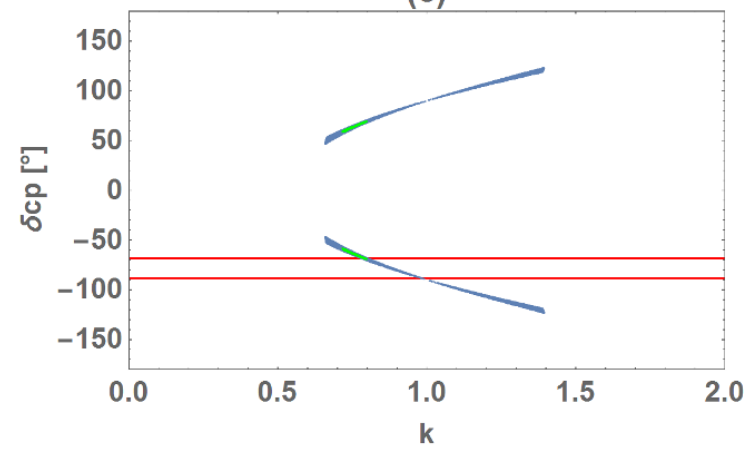

(b)

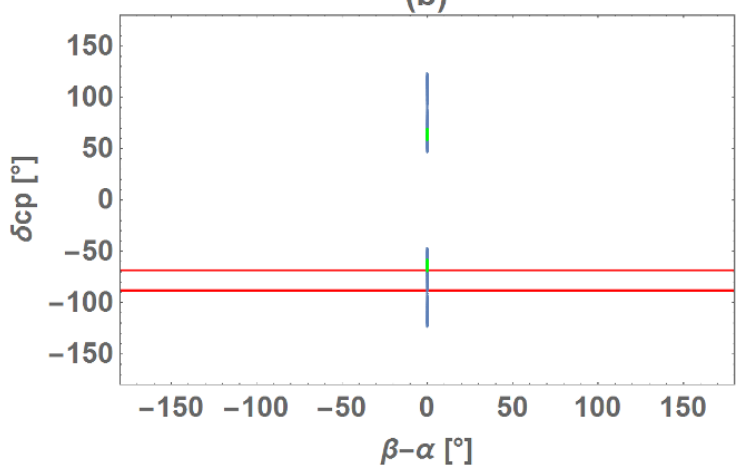

(d)

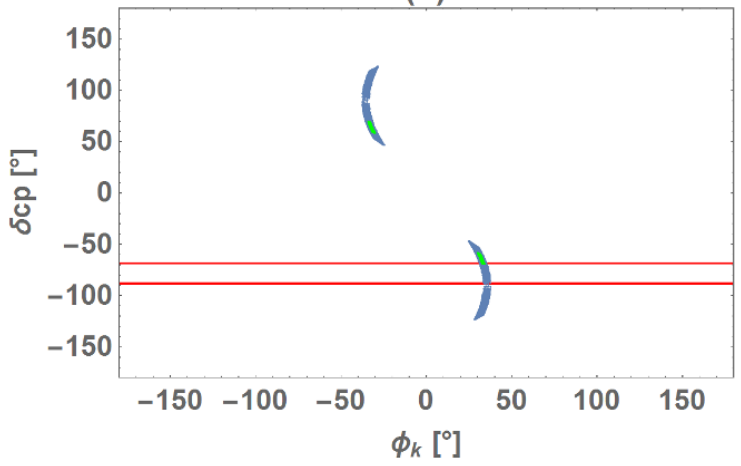

Figure 6. Blue (green) dots denote predictions for $\mathrm{TM}_{1}$ in $\mathrm{IH}$ with $3 \sigma(1 \sigma)$. The red lines for $\sin ^{2} \theta_{23}$ and $\delta_{\mathrm{CP}}$ denote the experimental bounds of $3 \sigma$ (global analyses) and $2 \sigma$ (T2K) ranges, respectively: (a) $\delta_{\mathrm{CP}}$ versus $\sin ^{2} \theta_{23}$, (b) $\delta_{\mathrm{CP}}$ versus difference of two Majorana phases $\beta-\alpha$, (c) $\delta_{\mathrm{CP}}$ versus $k$, and (d) $\delta_{\mathrm{CP}}$ versus $\phi_{k}$. The cyan dots in (a) denote the prediction of the two-zero texture [20].

In these results, we take $j=b / c$ to be real although the relative phase $b / c$ does not vanish in general as discussed below eq. (2.9). We have checked numerically that an extra phase of $b / c$ does not contribute the results in figure 5 while it affects the $\phi_{B}$ dependences of $\delta_{\mathrm{CP}}$ and $\left|m_{e e}\right|$ drastically. This situation is easily understood because the experimental data of mixing angles and mass square differences are input.

\section{3 $\mathrm{TM}_{1}$ : 2-3 family mixing in IH}

Although the case of the $\mathrm{NH}$ is rather favored in the experiments of $\mathrm{T} 2 \mathrm{~K}$ and $\mathrm{NO} \nu \mathrm{A}$ [2]-[4], the inverted hierarchy of neutrino masses is still allowed by the experimental data of the neutrino oscillations. Let us show the numerical result of the IH case $\left(m_{3}=0\right)$ for the 2-3 family mixing in eq. (2.24) since the Dirac neutrino mass matrix is completely different from the one of NH. In this case, the first column of the Dirac neutrino mass matrix is uniquely fixed as $(-2,1,1)^{\mathrm{T}}$. There are two free parameters, $k$ and $\phi_{k}$, as seen in eq. (2.23). In contrast to the $\mathrm{NH}$ case, the ratio $e / f$ is not allowed to be real in order to obtain the $\mathrm{CP}$ violation.

We show the predicted $\delta_{\mathrm{CP}}$ versus $\sin ^{2} \theta_{23}$ in figure $6(\mathrm{a})$. The prediction is same as the case $\mathrm{I}$ of $\mathrm{NH}$ in figure $1(\mathrm{a})$, that is $\pm\left(45^{\circ} \sim 125^{\circ}\right)$. The recent $\mathrm{T} 2 \mathrm{~K}$ data present $\delta_{\mathrm{CP}}$ 
to be in the range of $\left(-88^{\circ},-68^{\circ}\right)$ at $2 \sigma$ range for $\mathrm{IH}[3]$. We also show this range by red lines in the figures as an eye guide. We plot $\delta_{\mathrm{CP}}$ versus the difference of the two Majorana phases $\beta-\alpha$ in figure $6(\mathrm{~b})$. The difference of two Majorana phases $\beta-\alpha$ is almost zero. That is $\alpha=\beta$.

We also show the $k$ and $\phi_{k}$ dependencies of $\delta_{\mathrm{CP}}$ in figures $6(\mathrm{c})$ and $(\mathrm{d})$. The parameters are allowed in the narrow ranges $k=0.65 \sim 1.40$ and $\phi_{k}= \pm\left(25^{\circ} \sim 38^{\circ}\right)$. Thus, the structure of the Dirac neutrino mass matrix is restricted considerably.

The effective mass $\left|m_{e e}\right|$ is predicted to be around $50 \mathrm{meV}$ since $\beta-\alpha$ is almost zero and $\left|m_{e e}\right|$ does not depend on $\delta_{\mathrm{CP}}$ because of $m_{3}=0$.

In the context of the minimal seesaw model, the two-zero texture for the Dirac neutrino mass matrix has been examined in some works [15, 20, 21, 23]. This texture is different from our ones. However, this model is completely consistent with the experimental data of mixing angles and masses for $\mathrm{IH}$ [20] although this texture is disfavored for NH without some corrections. Since it is interesting to compare the predictions of two models, we add its prediction of $\delta_{\mathrm{CP}}$ in figures $6(\mathrm{a})$, where $(1,1)$ and $(2,2)$ elements of the $3 \times 2$ Dirac neutrino mass matrix are zero. The predicted $\delta_{\mathrm{CP}}$ is $\pm(88-93)^{\circ}$ in the whole range of allowed $\sin ^{2} \theta_{23}$. The case of vanishing $(1,1)$ and $(3,2)$ elements is also available, but the numerical prediction is almost unchanged. The prediction of the two-zero texture is a distinctive one from our ones.

\section{$3.4 \mathrm{TM}_{2}$ : 1-3 family mixing in $\mathrm{NH}$ or $\mathrm{IH}$}

In this subsection, we discuss the case of the 1-3 family mixing. As well known, the predicted $\sin ^{2} \theta_{12}$ is close to 0.34 [48], which is a characteristic one for $\mathrm{TM}_{2}$. The precise data of $\sin ^{2} \theta_{12}$ provides a crucial test for $\mathrm{TM}_{2}$ as well as $\mathrm{TM}_{1}$.

In figures 7 and 8, we show the numerical results for $\mathrm{NH}$ and $\mathrm{IH}$, respectively. For both $\mathrm{NH}$ and $\mathrm{IH}$ cases, $\delta_{\mathrm{CP}}$ is allowed to take all range in $(-\pi, \pi)$ as seen in figures 7 (a) and $8(\mathrm{a})$. It is found that the Dirac CP violating phase becomes maximal $\pm \pi / 2$ around the maximal mixing angle of $\theta_{23}=\pi / 4$. For $\mathrm{NH}$, the Majorana phase $\beta$ is allowed to take all values from $-\pi$ to $\pi$ as seen in figure $7(\mathrm{~b})$. On the other hand, for $\mathrm{IH}$, the difference of two Majorana phases $\beta-\alpha$ is almost zero as seen in figure $8(\mathrm{~b})$.

The predicted value of $\delta_{\mathrm{CP}}$ is sensitive to the parameter $k$ which behaves differently for $\mathrm{NH}$ and IH. In the case of $\mathrm{NH}, \delta_{\mathrm{CP}}$ is 0 or $\pm \pi$ at the lower bound of $k, 0.80$ or at the upper bound, 1.25, respectively, as seen in figure 7 (c). In the region of $k=0.80 \sim 1.25$, the predicted $\delta_{\mathrm{CP}}$ is changed drastically. On the other hand, for the case of $\mathrm{IH}, \delta_{\mathrm{CP}}$ is 0 or $\pm \pi$ at the lower bound $k=0.50$ or at the upper bound $k=2.00$, respectively, as seen in figure $8(\mathrm{c})$. In the region of $k=0.50 \sim 2.00, \delta_{\mathrm{CP}}$ is predicted for each $k$. The phase $\phi_{k}$ is restricted in the narrow region of $\pm\left(165^{\circ} \sim 180^{\circ}\right)$ for $\mathrm{NH}$ as seen in figure $7(\mathrm{~d})$. On the other hand, for $\mathrm{IH}$, the phase $\phi_{k}$ is in the region of $-37^{\circ} \sim 37^{\circ}$ as seen in figure $8(\mathrm{~d})$. In both cases, $\delta_{\mathrm{CP}}$ and $\theta_{23}$ become maximal simultaneously if $k=1$ is taken.

The effective mass $\left|m_{e e}\right|$ is predicted in the region around $1.6 \sim 4.2 \mathrm{meV}$ and $50 \mathrm{meV}$ for $\mathrm{NH}$ and $\mathrm{IH}$, respectively. 
(a)

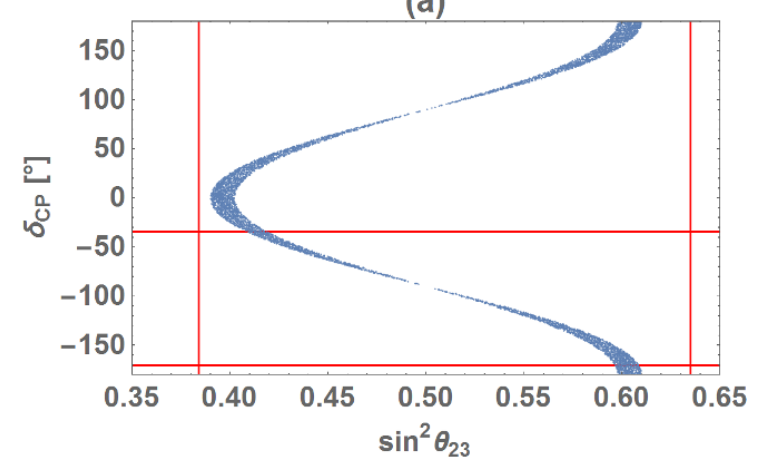

(c)

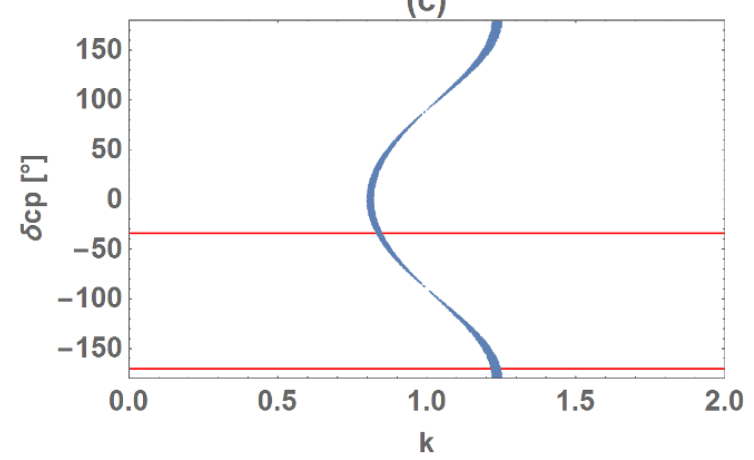

(b)

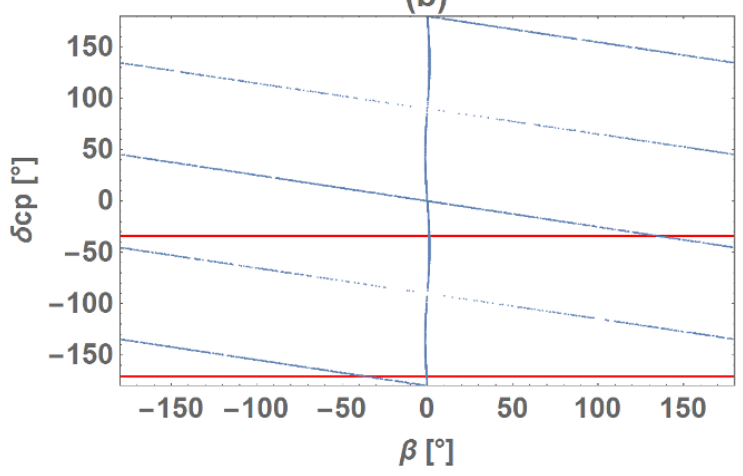

(d)

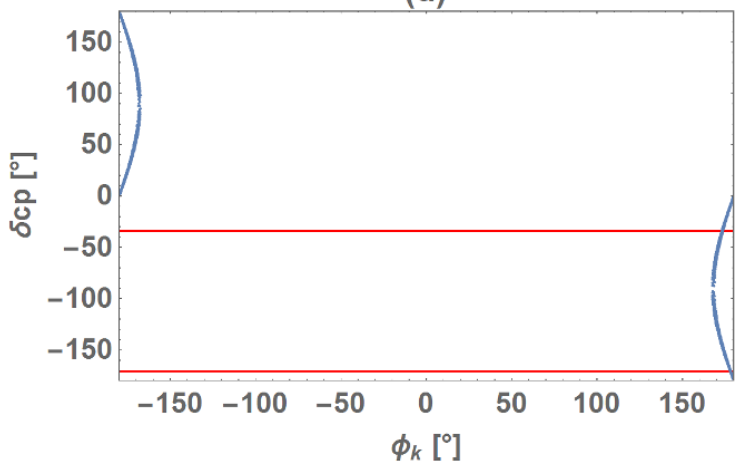

Figure 7. Predictions for $\mathrm{TM}_{2}$ in $\mathrm{NH}$, where the red lines for $\sin ^{2} \theta_{23}$ and $\delta_{\mathrm{CP}}$ denote the experimental bounds of $3 \sigma$ (global analyses) and $2 \sigma$ (T2K) ranges, respectively: (a) $\delta_{\mathrm{CP}}$ versus $\sin ^{2} \theta_{23}$, (b) $\delta_{\mathrm{CP}}$ versus Majorana phase $\beta$, (c) $\delta_{\mathrm{CP}}$ versus $k$, and (d) $\delta_{\mathrm{CP}}$ versus $\phi_{k}$.

\section{Summary and discussions}

We have studied the minimal seesaw model where only two right-handed Majorana neutrinos are assumed, focusing on the $\mathrm{CP}$ violating phase $\delta_{\mathrm{CP}}$. In addition, we have taken the trimaximal mixing pattern for the neutrino flavor $\left(\mathrm{TM}_{1}\right.$ or $\left.\mathrm{TM}_{2}\right)$ where the charged lepton mass matrix is diagonal. Owing to this symmetric framework, the flavor structure of the $3 \times 2$ Dirac neutrino mass matrix is given in terms of a few parameters.

We have examined three cases of the Dirac neutrino mass matrix for $\mathrm{TM}_{1}$ in NH. It is emphasized that the observation of the $\mathrm{CP}$ violating phase determines the flavor structure of the Dirac neutrino mass matrix in the minimal seesaw model. New minimal Dirac neutrino mass matrices have been presented as the result of the numerical study for case I. Our model includes the Littlest seesaw model by King et al. as the one of the specific cases. We have also discussed the case of $\mathrm{TM}_{1}$ in IH. The structure of the Dirac neutrino mass matrix is restricted considerably. The parameters are determined in the narrow ranges, $k=0.65 \sim 1.40$ and $\phi_{k}= \pm\left(25^{\circ} \sim 38^{\circ}\right)$. We have studied the case of $\mathrm{TM}_{2}$ in $\mathrm{NH}$ and IH. The predicted $\delta_{\mathrm{CP}}$ is allowed to take all range in $(-\pi, \pi)$, but it is sensitive to the parameter $k$, which is around 1 . In both cases, $\delta_{\mathrm{CP}}$ and the mixing angle $\theta_{23}$ become maximal simultaneously for $k=1$. 
(a)

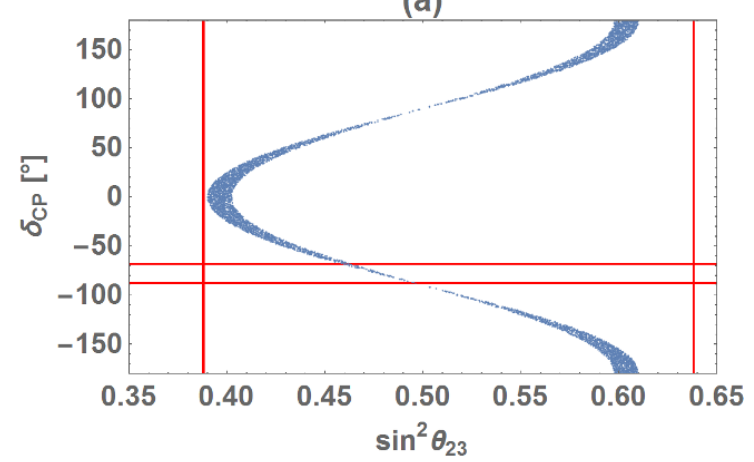

(c)

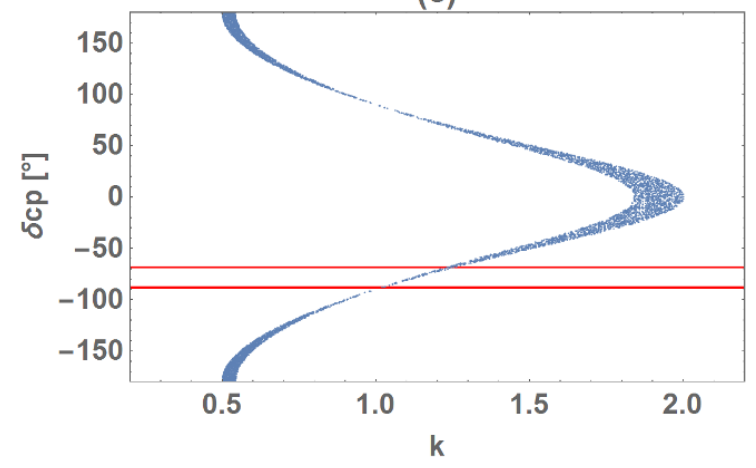

(b)

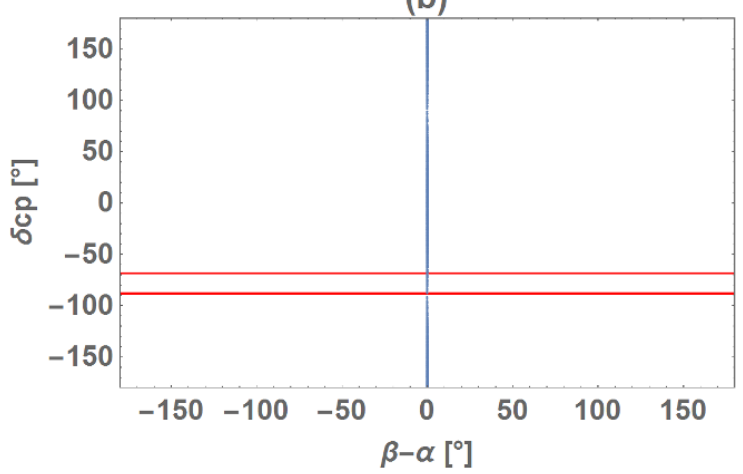

(d)

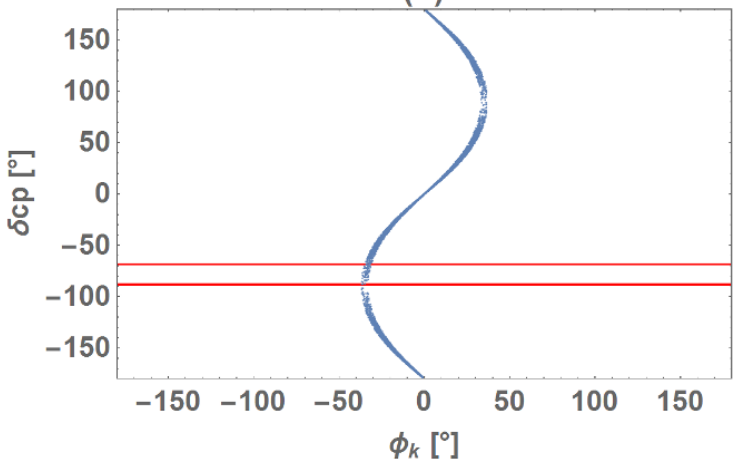

Figure 8. Predictions for $\mathrm{TM}_{2}$ in $\mathrm{IH}$, where the red lines for $\sin ^{2} \theta_{23}$ and $\delta_{\mathrm{CP}}$ denote the experimental bounds of $3 \sigma$ (global analyses) and $2 \sigma$ (T2K), respectively: (a) $\delta_{\mathrm{CP}}$ versus $\sin ^{2} \theta_{23}$, (b) $\delta_{\mathrm{CP}}$ versus difference of two Majorana phases $\beta-\alpha$, (c) $\delta_{\mathrm{CP}}$ versus $k$, and (d) $\delta_{\mathrm{CP}}$ versus $\phi_{k}$.

Our $3 \times 2$ Dirac neutrino mass matrix is reproduced by introducing gauge singlet flavons with the VEV's in $S_{4}$ flavor symmetry. The specific alignments of the VEV's are derived from the residual symmetry of $S_{4}$ group. It is interesting to consider the underlying mechanism which dynamically realizes the required vacuum alignments.

Finally, we add comments. If our Dirac neutrino mass matrices are given at the high energy scale, for example, the GUT scale, one should examine the renormalization group correction for the neutrino mixing matrix. However, it is very small since the lightest neutrino mass vanishes in our framework as seen in ref. [64].

The sign of the $\mathrm{CP}$ violating phase $\delta_{\mathrm{CP}}$ is not determined in our framework. As well known, $\mathrm{CP}$ violating phases in the neutrino mass matrix are related to $\mathrm{CP}$ violating phases at the high energy. Since one can discuss the baryon asymmetry of the universe assuming the leptogenesis [65], the sign of $\delta_{\mathrm{CP}}$ can be predicted in our framework. This work will appear elsewhere.

\section{Acknowledgments}

We thank Akihiro Yu for careful reading of the manuscript. This work is supported by JSPS Grants-in-Aid for Scientific Research 16J05332 (YS) and 15K05045, $16 \mathrm{H} 00862$ (MT). 


\section{A Minimal seesaw mass matrix}

We can take the $2 \times 2$ right-handed Majorana neutrino mass matrix $M_{R}$ to be real diagonal in general:

$$
M_{R}=-M_{0}\left(\begin{array}{cc}
p^{-1} & 0 \\
0 & 1
\end{array}\right),
$$

where $M_{0}$ is the mass scale of the right-handed Majorana neutrino and $p$ is the ratio between the two right-handed Majorana neutrino masses. The minus sign in front of $M_{0}$ is taken as our sign convention. On the other hand, the relevant Dirac neutrino mass matrix $M_{D}$ is defined as

$$
M_{D}=\left(\begin{array}{ll}
a & d \\
b & e \\
c & f
\end{array}\right),
$$

where $a \sim f$ are complex parameters. By using the seesaw mechanism, the left-handed Majorana neutrino mass matrix $M_{\nu}$ is given by

$$
M_{\nu}=M_{D} M_{R}^{-1} M_{D}^{T}=\frac{1}{M_{0}}\left(\begin{array}{ccc}
a^{2} p+d^{2} & a b p+d e & a c p+d f \\
a b p+d e & b^{2} p+e^{2} & b c p+e f \\
a c p+d f & b c p+e f & c^{2} p+f^{2}
\end{array}\right) .
$$

By turning the neutrino mass matrix $M_{\nu}$ to the TBM mixing basis, $M_{\nu}$ is given as

$$
\hat{M}_{\nu} \equiv V_{\mathrm{TBM}}^{T} M_{\nu} V_{\mathrm{TBM}}=\frac{1}{M_{0}}\left(\begin{array}{ccc}
\frac{A_{\nu}^{2} p+D_{\nu}^{2}}{6} & \frac{A_{\nu} B_{\nu} p+D_{\nu} E_{\nu}}{3 \sqrt{2}} & \frac{A_{\nu} C_{\nu} p+D_{\nu} F_{\nu}}{2 \sqrt{3}} \\
\frac{A_{\nu} B_{\nu} p+D_{\nu} E_{\nu}}{3 \sqrt{2}} & \frac{B_{\nu}^{2} p+E_{\nu}^{2}}{3} & \frac{B_{\nu} C_{\nu} p+E_{\nu} F_{\nu}}{\sqrt{6}} \\
\frac{A_{\nu} C_{\nu} p+D_{\nu} F_{\nu}}{2 \sqrt{3}} & \frac{B_{\nu} C_{\nu} p+E_{\nu} F_{\nu}}{\sqrt{6}} & \frac{C_{\nu}^{2} p+F_{\nu}^{2}}{2}
\end{array}\right),
$$

where

$$
\begin{array}{rlrl}
A_{\nu} & \equiv 2 a-b-c, & B_{\nu} \equiv a+b+c, & C_{\nu} \equiv c-b, \\
D_{\nu} \equiv 2 d-e-f, & E_{\nu} \equiv d+e+f, & F_{\nu} \equiv f-e .
\end{array}
$$

We discuss neutrino mass structures to realize the additional 2-3 family rotation $\left(\mathrm{TM}_{1}\right)$ and the additional 1-3 one $\left(\mathrm{TM}_{2}\right)$ to the TBM mixing basis for both $\mathrm{NH}$ and $\mathrm{IH}$.

\section{A.1 $\mathrm{TM}_{1}$ : additional $2-3$ family rotation in $\mathrm{NH}$}

At first, we consider the case of $\mathrm{NH}$ in $\mathrm{TM}_{1}$. Since $(1,1),(1,2),(2,1),(1,3)$, and $(3,1)$ entries of the matrix must be zero, conditions for the additional 2-3 rotation to the TBM mixing are

$$
A_{\nu}=2 a-b-c=0, \quad D_{\nu}=2 d-e-f=0,
$$

where $(a, b, c)$ are supposed to be independent of $(d, e, f)$. After imposing these conditions on the eq. (A.4), the mass matrix is rewritten as

$$
\hat{M}_{\nu}=\frac{1}{M_{0}}\left(\begin{array}{ccc}
0 & 0 & 0 \\
0 & \frac{3}{4}\left((b+c)^{2} p+(e+f)^{2}\right) & \frac{1}{2} \sqrt{\frac{3}{2}}\left(\left(c^{2}-b^{2}\right) p-e^{2}+f^{2}\right) \\
0 & \frac{1}{2} \sqrt{\frac{3}{2}}\left(\left(c^{2}-b^{2}\right) p-e^{2}+f^{2}\right) & \frac{1}{2}\left((b-c)^{2} p+(e-f)^{2}\right)
\end{array}\right),
$$


where the lightest neutrino mass $m_{1}$ is zero. Since the Majorana neutrino mass can be rescaled in the seesaw formula, the right-handed Majorana and Dirac neutrino mass matrices are written by putting $p=1$ as

$$
M_{R}=-M_{0}\left(\begin{array}{ll}
1 & 0 \\
0 & 1
\end{array}\right), \quad M_{D}=\left(\begin{array}{cc}
\frac{b+c}{2} & \frac{e+f}{2} \\
b & e \\
c & f
\end{array}\right)
$$

respectively. Starting from these textures, we discuss the specific cases which are attractive in the standpoint of the flavor model.

We consider one zero textures leading to the additional 2-3 family rotation to the TBM mixing basis. There are three possible patterns of one zero texture as follows:

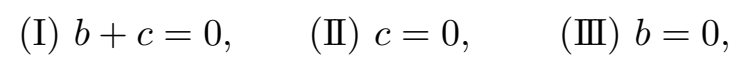

in eq. (A.8). Corresponding Dirac neutrino mass matrices can be obtained as

$$
M_{D}= \begin{cases}\left(\begin{array}{cc}
0 & \frac{e+f}{2} \\
b & e \\
-b & f
\end{array}\right) & \text { for (I) } b+c=0 \\
\left(\begin{array}{cc}
\frac{b}{2} & \frac{e+f}{2} \\
b & e \\
0 & f \\
\frac{c}{2} & \frac{e+f}{2} \\
0 & e \\
c & f
\end{array}\right) & \text { for (II) } c=0 \\
\text { for (III) } b=0\end{cases}
$$

One can get another set by exchanging between the first column and second one in the Dirac neutrino mass matrix of eq. (A.10). However, the neutrino mass matrix $\hat{M}_{\nu}$ is invariant by this exchange. Therefore, we consider only three cases in eq. (A.10).

We show the neutrino mass matrix $\hat{M}_{\nu}$ for three cases:

Case I : $\quad \hat{M}_{\nu}=\frac{1}{M_{0}}\left(\begin{array}{ccc}0 & 0 & 0 \\ 0 & \frac{3}{4}(e+f)^{2} & -\frac{1}{2} \sqrt{\frac{3}{2}}(e-f)(e+f) \\ 0-\frac{1}{2} \sqrt{\frac{3}{2}}(e-f)(e+f) & 2 b^{2}+\frac{1}{2}(e-f)^{2}\end{array}\right)$,

Case II : $\quad \hat{M}_{\nu}=\frac{1}{M_{0}}\left(\begin{array}{ccc}0 & 0 & 0 \\ 0 & \frac{3}{4}\left[b^{2}+(e+f)^{2}\right] & -\frac{1}{2} \sqrt{\frac{3}{2}}\left[b^{2}+(e-f)(e+f)\right] \\ 0-\frac{1}{2} \sqrt{\frac{3}{2}}\left[b^{2}+(e-f)(e+f)\right] & \frac{1}{2}\left[b^{2}+(e-f)^{2}\right]\end{array}\right)$,

Case III $: \hat{M}_{\nu}=\frac{1}{M_{0}}\left(\begin{array}{ccc}0 & 0 & 0 \\ 0 & \frac{3}{4}\left[c^{2}+(e+f)^{2}\right] & -\frac{1}{2} \sqrt{\frac{3}{2}}\left[-c^{2}+(e-f)(e+f)\right] \\ 0-\frac{1}{2} \sqrt{\frac{3}{2}}\left[-c^{2}+(e-f)(e+f)\right] & \frac{1}{2}\left[c^{2}+(e-f)^{2}\right]\end{array}\right)$. 


\section{A.2 $\mathrm{TM}_{1}$ : additional $2-3$ rotation in $\mathrm{IH}$}

Let us discuss the case of $\mathrm{IH}$ in $\mathrm{TM}_{1}$. In order to give the additional 2-3 family rotation to the TBM mixing, the $(1,2),(1,3),(2,1)$, and $(3,1)$ elements in eq. (A.4) should vanish. These conditions are given as

$$
A_{\nu}=a+b+c=0, \quad C_{\nu}=c-b=0, \quad D_{\nu}=2 d-e-f=0 .
$$

By setting $p=1$, we have

$$
M_{R}=-M_{0}\left(\begin{array}{ll}
1 & 0 \\
0 & 1
\end{array}\right), \quad M_{D}=\left(\begin{array}{cc}
-2 b & \frac{e+f}{2} \\
b & e \\
b & f
\end{array}\right) .
$$

The neutrino mass matrix $\hat{M}_{\nu}$ is given as

$$
\hat{M}_{\nu}=\frac{1}{M_{0}}\left(\begin{array}{ccc}
6 b^{2} & 0 & 0 \\
0 & \frac{3}{4}(e+f)^{2} & -\frac{1}{2} \sqrt{\frac{3}{2}}(e-f)(e+f) \\
0 & -\frac{1}{2} \sqrt{\frac{3}{2}}(e-f)(e+f) & \frac{1}{2}(e-f)^{2}
\end{array}\right),
$$

where the neutrino mass $m_{3}$ vanishes.

\section{A.3 $\mathrm{TM}_{2}$ : additional 1-3 rotation in $\mathrm{NH}$ or $\mathrm{IH}$}

Let us consider the case of the additional 1-3 family rotation to the TBM mixing basis. This case is called as $\mathrm{TM}_{2}$. Then, $(1,2),(2,3),(2,1)$, and $(3,2)$ elements in eq. (A.4) should vanish. These conditions are given as

$$
A_{\nu}=2 a-b-c=0, \quad C_{\nu}=c-b=0, \quad E_{\nu}=d+e+f=0 .
$$

The neutrino mass matrix $\hat{M}_{\nu}$ is written by

$$
\hat{M}_{\nu}=\frac{1}{M_{0}}\left(\begin{array}{ccc}
\frac{3}{2}(e+f)^{2} & 0 & \frac{\sqrt{3}}{2}\left(e^{2}-f^{2}\right) \\
0 & 3 b^{2} & 0 \\
\frac{\sqrt{3}}{2}\left(e^{2}-f^{2}\right) & 0 & \frac{1}{2}(e-f)^{2}
\end{array}\right),
$$

where $p=1$ is set. The right-handed Majorana and the Dirac neutrino mass matrices are

$$
M_{R}=-M_{0}\left(\begin{array}{ll}
1 & 0 \\
0 & 1
\end{array}\right), \quad M_{D}=\left(\begin{array}{cc}
b-e-f \\
b & e \\
b & f
\end{array}\right),
$$

respectively. There is another solution

$$
B_{\nu}=a+b+c=0, \quad D_{\nu}=2 d-e-f=0, \quad F_{\nu}=f-e=0 .
$$

However, this set leads to same structure of the neutrino mass matrix as in eq. (A.18). The mass eigenvalue $m_{1}$ or $m_{3}$ vanishes for $\mathrm{NH}$ or $\mathrm{IH}$, respectively. 


\section{B Representations of $S_{4}$ group}

We show the representations of $S_{4}$ group in this appendix. All elements of $S_{4}$ group are expressed by products of the generators $s$ and $t$, which satisfy

$$
s^{4}=t^{3}=e, \quad s t^{2} s=t, \quad s t s=t s^{2} t,
$$

where $e$ is an identity element. These generators are represented on $\mathbf{1}_{\mathbf{1}}, \mathbf{1}_{\mathbf{2}}, \mathbf{2}, \mathbf{3}_{\mathbf{1}}$, and $\mathbf{3}_{\mathbf{2}}$ of $S_{4}$ group as follows [36, 37]:

$$
\begin{array}{rlrl}
\mathbf{1}_{\mathbf{1}}: & s=1, & t & =1, \\
\mathbf{1}_{\mathbf{2}}: & s=-1, & t=1, & \\
\mathbf{2}: & s=\left(\begin{array}{ll}
0 & 1 \\
1 & 0
\end{array}\right), & t & =\left(\begin{array}{cc}
\omega & 0 \\
0 & \omega^{2}
\end{array}\right), \\
\mathbf{3}_{\mathbf{1}}: & s=\frac{1}{3}\left(\begin{array}{ccc}
-1 & 2 \omega & 2 \omega^{2} \\
2 \omega & 2 \omega^{2} & -1 \\
2 \omega^{2} & -1 & 2 \omega
\end{array}\right), & t=\left(\begin{array}{ccc}
1 & 0 & 0 \\
0 & \omega^{2} & 0 \\
0 & 0 & \omega
\end{array}\right), \\
\mathbf{3}_{\mathbf{2}}: & s=-\frac{1}{3}\left(\begin{array}{ccc}
-1 & 2 \omega & 2 \omega^{2} \\
2 \omega & 2 \omega^{2} & -1 \\
2 \omega^{2} & -1 & 2 \omega
\end{array}\right), & t=\left(\begin{array}{ccc}
1 & 0 & 0 \\
0 & \omega^{2} & 0 \\
0 & 0 & \omega
\end{array}\right) .
\end{array}
$$

On the other hand, in refs. [66, 67], all elements of $S_{4}$ group are also expressed by products of the three generators $S, T$, and $U$, which satisfy

$$
S^{2}=T^{3}=U^{2}=(S T)^{3}=(S U)^{2}=(T U)^{2}=(S T U)^{4}=e .
$$

Note that the minimal number of $S_{4}$ generators is only two. However, in order to compare generators of $S_{4}$ group with that of $A_{4}$ group, it is convenient to express elements of the group in terms of $S, T$, and $U$. These generators are also represented on $\mathbf{1}, \mathbf{1}^{\prime}, \mathbf{2}, \mathbf{3}$, and $\mathbf{3}^{\prime}$ of $S_{4}$ group as follows:

$$
\begin{array}{llll}
\mathbf{1}: S=1, & T=1, & U=1, \\
\mathbf{1}^{\prime}: & S=1, & T=1, & U=-1, \\
\mathbf{2}: & S=\left(\begin{array}{ll}
1 & 0 \\
0 & 1
\end{array}\right), & T=\left(\begin{array}{cc}
\omega & 0 \\
0 & \omega^{2}
\end{array}\right), & U=\left(\begin{array}{ll}
0 & 1 \\
1 & 0
\end{array}\right), \\
\mathbf{3}: S=\frac{1}{3}\left(\begin{array}{ccc}
-1 & 2 & 2 \\
2 & -1 & 2 \\
2 & 2 & -1
\end{array}\right), & T=\left(\begin{array}{ccc}
1 & 0 & 0 \\
0 & \omega^{2} & 0 \\
0 & 0 & \omega
\end{array}\right), & U=-\left(\begin{array}{lll}
1 & 0 & 0 \\
0 & 0 & 1 \\
0 & 1 & 0
\end{array}\right), \\
\mathbf{3}^{\prime}: & S=\frac{1}{3}\left(\begin{array}{ccc}
-1 & 2 & 2 \\
2 & -1 & 2 \\
2 & 2 & -1
\end{array}\right), & T=\left(\begin{array}{lll}
1 & 0 & 0 \\
0 & \omega^{2} & 0 \\
0 & 0 & \omega
\end{array}\right), & U=\left(\begin{array}{lll}
1 & 0 & 0 \\
0 & 0 & 1 \\
0 & 1 & 0
\end{array}\right),
\end{array}
$$


where $\mathbf{1}$ corresponds to $\mathbf{1}_{\mathbf{1}}, \mathbf{1}^{\prime}$ corresponds to $\mathbf{1}_{\mathbf{2}}, \mathbf{3}$ corresponds to $\mathbf{3}_{\mathbf{2}}$, and $\mathbf{3}^{\prime}$ corresponds to $\mathbf{3}_{\mathbf{1}}$ in eq. (B.2), respectively. It is remarked that $s$ and $t$ generators are related with $S$, $T$, and $U$ as follows:

$$
s=S T U S T, \quad t=T .
$$

The tensor products of $S_{4}$ group are independent of the basis of representations and written as follows:

$$
\begin{aligned}
\mathbf{1} \otimes \mathbf{r} & =\mathbf{r} \otimes \mathbf{1}=\mathbf{r}, & \mathbf{1}^{\prime} \otimes \mathbf{1}^{\prime} & =\mathbf{1}, \\
\mathbf{1}^{\prime} \otimes \mathbf{3} & =\mathbf{3} \otimes \mathbf{1}^{\prime}=\mathbf{3}^{\prime}, & \mathbf{1}^{\prime} \otimes \mathbf{3}^{\prime} & =\mathbf{3}^{\prime} \otimes \mathbf{1}^{\prime}=\mathbf{3}, \\
\mathbf{2} \otimes \mathbf{2} & =\mathbf{1} \oplus \mathbf{1}^{\prime} \oplus \mathbf{2}, & \mathbf{2} \otimes \mathbf{3} & =\mathbf{3} \otimes \mathbf{2}=\mathbf{2} \otimes \mathbf{3}^{\prime}=\mathbf{3}^{\prime} \otimes \mathbf{2}=\mathbf{3} \oplus \mathbf{1}^{\prime}, \\
\mathbf{3} \otimes \mathbf{3} & =\mathbf{3}^{\prime} \otimes \mathbf{3}^{\prime}=\mathbf{1} \oplus \mathbf{2} \oplus \mathbf{3} \oplus \mathbf{3}^{\prime}, & \mathbf{3} \otimes \mathbf{3}^{\prime} & =\mathbf{3}^{\prime} \otimes \mathbf{3}=\mathbf{1}^{\prime} \oplus \mathbf{2} \oplus \mathbf{3} \oplus \mathbf{3}^{\prime},
\end{aligned}
$$

where $\mathbf{r}$ is an arbitrary representation of $S_{4}$ group.

For the following tensor products,

$$
\mathbf{3} \otimes \mathbf{3} \rightarrow \mathbf{1}, \quad \mathbf{3}^{\prime} \otimes 3^{\prime} \rightarrow \mathbf{1}, \quad \mathbf{3} \otimes \mathbf{3}^{\prime} \rightarrow \mathbf{1}^{\prime},
$$

the Clebsch-Gordan coefficient is given as

$$
\alpha_{1} \beta_{1}+\alpha_{2} \beta_{3}+\alpha_{3} \beta_{2}
$$

where $\alpha_{i}$ and $\beta_{i}$ are elements of $\mathbf{3}^{\left({ }^{\prime}\right)}$.

Open Access. This article is distributed under the terms of the Creative Commons Attribution License (CC-BY 4.0), which permits any use, distribution and reproduction in any medium, provided the original author(s) and source are credited.

\section{References}

[1] T2K collaboration, K. Abe et al., Observation of Electron Neutrino Appearance in a Muon Neutrino Beam, Phys. Rev. Lett. 112 (2014) 061802 [arXiv:1311.4750] [INSPIRE].

[2] T2K collaboration, K. Abe et al., Measurement of neutrino and antineutrino oscillations by the T2K experiment including a new additional sample of $\nu_{e}$ interactions at the far detector, Phys. Rev. D 96 (2017) 092006 [arXiv:1707.01048] [INSPIRE].

[3] T2K collaboration, T2K presents hint of CP violation by neutrinos, T2K report, 4 August 2017, http://t2k-experiment.org/2017/08/t2k-2017-cpv/.

[4] NOvA collaboration, P. Adamson et al., Constraints on Oscillation Parameters from $\nu_{e}$ Appearance and $\nu_{\mu}$ Disappearance in NOvA, Phys. Rev. Lett. 118 (2017) 231801 [arXiv: 1703.03328] [INSPIRE].

[5] P. Minkowski, $\mu \rightarrow$ e $\gamma$ at a Rate of One Out of $10^{9}$ Muon Decays?, Phys. Lett. B 67 (1977) 421 [INSPIRE].

[6] T. Yanagida, Horizontal symmetry and masses of neutrinos, Conf. Proc. C 7902131 (1979) 95 [INSPIRE].

[7] M. Gell-Mann, P. Ramond and R. Slansky, Complex Spinors and Unified Theories, Conf. Proc. C 790927 (1979) 315 [arXiv:1306.4669] [INSPIRE]. 
[8] R.N. Mohapatra and G. Senjanović, Neutrino Mass and Spontaneous Parity Violation, Phys. Rev. Lett. 44 (1980) 912 [INSPIRE].

[9] J. Schechter and J.W.F. Valle, Neutrino Masses in $\mathrm{SU}(2) \times \mathrm{U}(1)$ Theories, Phys. Rev. D 22 (1980) 2227 [INSPIRE].

[10] J. Schechter and J.W.F. Valle, Neutrino Decay and Spontaneous Violation of Lepton Number, Phys. Rev. D 25 (1982) 774 [INSPIRE].

[11] Y. Shimizu, R. Takahashi and M. Tanimoto, Minimal Neutrino Texture with Neutrino Mass Ratio and Cabibbo Angle, PTEP 2013 (2013) 063B02 [arXiv:1212.5913] [INSPIRE].

[12] S.F. King, Atmospheric and solar neutrinos with a heavy singlet, Phys. Lett. B 439 (1998) 350 [hep-ph/9806440] [INSPIRE].

[13] S.F. King, Atmospheric and solar neutrinos from single right-handed neutrino dominance and U(1) family symmetry, Nucl. Phys. B 562 (1999) 57 [hep-ph/9904210] [INSPIRE].

[14] G.C. Branco, R. Gonzalez Felipe, F.R. Joaquim and T. Yanagida, Removing ambiguities in the neutrino mass matrix, Phys. Lett. B 562 (2003) 265 [hep-ph/0212341] [INSPIRE].

[15] P.H. Frampton, S.L. Glashow and T. Yanagida, Cosmological sign of neutrino CP-violation, Phys. Lett. B 548 (2002) 119 [hep-ph/0208157] [InSPIRE].

[16] K. Bhattacharya, N. Sahu, U. Sarkar and S.K. Singh, Leptogenesis and low energy CP phases with two heavy neutrinos, Phys. Rev. D 74 (2006) 093001 [hep-ph/0607272] [INSPIRE].

[17] S. Goswami and A. Watanabe, Minimal Seesaw Textures with Two Heavy Neutrinos, Phys. Rev. D 79 (2009) 033004 [arXiv:0807.3438] [INSPIRE].

[18] S. Goswami, S. Khan and A. Watanabe, Hybrid textures in minimal seesaw mass matrices, Phys. Lett. B 693 (2010) 249 [arXiv:0811.4744] [InSPIRE].

[19] W. Rodejohann, M. Tanimoto and A. Watanabe, Relating large $U_{e 3}$ to the ratio of neutrino mass-squared differences, Phys. Lett. B 710 (2012) 636 [arXiv:1201.4936] [INSPIRE].

[20] K. Harigaya, M. Ibe and T.T. Yanagida, Seesaw Mechanism with Occam's Razor, Phys. Rev. D 86 (2012) 013002 [arXiv: 1205.2198] [INSPIRE].

[21] J. Zhang and S. Zhou, A Further Study of the Frampton-Glashow-Yanagida Model for Neutrino Masses, Flavor Mixing and Baryon Number Asymmetry, JHEP 09 (2015) 065 [arXiv: 1505.04858] [INSPIRE].

[22] G. Bambhaniya, P. Bhupal Dev, S. Goswami, S. Khan and W. Rodejohann, Naturalness, Vacuum Stability and Leptogenesis in the Minimal Seesaw Model, Phys. Rev. D 95 (2017) 095016 [arXiv: 1611.03827] [INSPIRE].

[23] T. Rink and K. Schmitz, Perturbed Yukawa Textures in the Minimal Seesaw Model, JHEP 03 (2017) 158 [arXiv:1611.05857] [INSPIRE].

[24] T. Rink, K. Schmitz and T.T. Yanagida, Minimal Seesaw Model with a Discrete Heavy-Neutrino Exchange Symmetry, arXiv:1612.08878 [INSPIRE].

[25] W. Grimus and L. Lavoura, A model for trimaximal lepton mixing, JHEP 09 (2008) 106 [arXiv: 0809.0226] [INSPIRE].

[26] C.H. Albright and W. Rodejohann, Comparing Trimaximal Mixing and Its Variants with Deviations from Tri-bimaximal Mixing, Eur. Phys. J. C 62 (2009) 599 [arXiv:0812.0436] [INSPIRE]. 
[27] DAYA BAy collaboration, F.P. An et al., Observation of electron-antineutrino disappearance at Daya Bay, Phys. Rev. Lett. 108 (2012) 171803 [arXiv:1203.1669] [InSPIRE].

[28] RENO collaboration, J.K. Ahn et al., Observation of Reactor Electron Antineutrino Disappearance in the RENO Experiment, Phys. Rev. Lett. 108 (2012) 191802 [arXiv: 1204.0626] [INSPIRE].

[29] P.F. Harrison, D.H. Perkins and W.G. Scott, Tri-bimaximal mixing and the neutrino oscillation data, Phys. Lett. B 530 (2002) 167 [hep-ph/0202074] [INSPIRE].

[30] P.F. Harrison and W.G. Scott, Symmetries and generalizations of tri - bimaximal neutrino mixing, Phys. Lett. B 535 (2002) 163 [hep-ph/0203209] [INSPIRE].

[31] E. Ma and G. Rajasekaran, Softly broken $A_{4}$ symmetry for nearly degenerate neutrino masses, Phys. Rev. D 64 (2001) 113012 [hep-ph/0106291] [INSPIRE].

[32] K.S. Babu, E. Ma and J.W.F. Valle, Underlying $A_{4}$ symmetry for the neutrino mass matrix and the quark mixing matrix, Phys. Lett. B 552 (2003) 207 [hep-ph/0206292] [INSPIRE].

[33] G. Altarelli and F. Feruglio, Tri-bimaximal neutrino mixing from discrete symmetry in extra dimensions, Nucl. Phys. B 720 (2005) 64 [hep-ph/0504165] [InSPIRE].

[34] G. Altarelli and F. Feruglio, Tri-bimaximal neutrino mixing, $A_{4}$ and the modular symmetry, Nucl. Phys. B 741 (2006) 215 [hep-ph/0512103] [INSPIRE].

[35] G. Altarelli and F. Feruglio, Discrete Flavor Symmetries and Models of Neutrino Mixing, Rev. Mod. Phys. 82 (2010) 2701 [arXiv: 1002.0211] [InSPIRE].

[36] H. Ishimori, T. Kobayashi, H. Ohki, Y. Shimizu, H. Okada and M. Tanimoto, Non-Abelian Discrete Symmetries in Particle Physics, Prog. Theor. Phys. Suppl. 183 (2010) 1 [arXiv:1003.3552] [INSPIRE].

[37] H. Ishimori, T. Kobayashi, H. Ohki, H. Okada, Y. Shimizu and M. Tanimoto, An introduction to non-Abelian discrete symmetries for particle physicists, Lect. Notes Phys. 858 (2012) 1 [INSPIRE].

[38] S.F. King, A. Merle, S. Morisi, Y. Shimizu and M. Tanimoto, Neutrino Mass and Mixing: from Theory to Experiment, New J. Phys. 16 (2014) 045018 [arXiv:1402.4271] [INSPIRE].

[39] C.H. Albright, A. Dueck and W. Rodejohann, Possible Alternatives to Tri-bimaximal Mixing, Eur. Phys. J. C 70 (2010) 1099 [arXiv:1004.2798] [INSPIRE].

[40] W. Rodejohann and H. Zhang, Simple two Parameter Description of Lepton Mixing, Phys. Rev. D 86 (2012) 093008 [arXiv:1207.1225] [INSPIRE].

[41] S. Antusch, S.F. King, C. Luhn and M. Spinrath, Trimaximal mixing with predicted $\theta_{13}$ from a new type of constrained sequential dominance, Nucl. Phys. B 856 (2012) 328 [arXiv:1108.4278] [INSPIRE].

[42] P. Ballett, S.F. King, C. Luhn, S. Pascoli and M.A. Schmidt, Testing atmospheric mixing sum rules at precision neutrino facilities, Phys. Rev. D 89 (2014) 016016 [arXiv:1308.4314] [INSPIRE].

[43] S.T. Petcov, Predicting the values of the leptonic CP-violation phases in theories with discrete flavour symmetries, Nucl. Phys. B 892 (2015) 400 [arXiv: 1405.6006] [INSPIRE].

[44] D. Marzocca, S.T. Petcov, A. Romanino and M.C. Sevilla, Nonzero $\left|U_{e} 3\right|$ from Charged Lepton Corrections and the Atmospheric Neutrino Mixing Angle, JHEP 05 (2013) 073 [arXiv:1302.0423] [INSPIRE]. 
[45] Z.-z. Xing and S. Zhou, A partial $\mu-\tau$ symmetry and its prediction for leptonic CP-violation, Phys. Lett. B 737 (2014) 196 [arXiv:1404.7021] [InSPIRE].

[46] G.C. Branco, M.N. Rebelo, J.I. Silva-Marcos and D. Wegman, Quasidegeneracy of Majorana Neutrinos and the Origin of Large Leptonic Mixing, Phys. Rev. D 91 (2015) 013001 [arXiv:1405.5120] [INSPIRE].

[47] S.K. Kang and C.S. Kim, Prediction of leptonic CP phase from perturbatively modified tribimaximal (or bimaximal) mixing, Phys. Rev. D 90 (2014) 077301 [arXiv:1406.5014] [INSPIRE].

[48] Y. Shimizu, M. Tanimoto and K. Yamamoto, Predicting CP-violation in Deviation from Tri-bimaximal mixing of Neutrinos, Mod. Phys. Lett. A 30 (2015) 1550002 [arXiv: 1405.1521] [INSPIRE].

[49] S.K. Kang and M. Tanimoto, Prediction of Leptonic CP Phase in $A_{4}$ symmetric model, Phys. Rev. D 91 (2015) 073010 [arXiv:1501.07428] [INSPIRE].

[50] I. Girardi, S.T. Petcov and A.V. Titov, Determining the Dirac CP-violation Phase in the Neutrino Mixing Matrix from Sum Rules, Nucl. Phys. B 894 (2015) 733 [arXiv:1410.8056] [INSPIRE].

[51] I. Girardi, S.T. Petcov and A.V. Titov, Predictions for the Leptonic Dirac CP-violation Phase: a Systematic Phenomenological Analysis, Eur. Phys. J. C 75 (2015) 345 [arXiv: 1504.00658] [INSPIRE].

[52] I. Girardi, S.T. Petcov, A.J. Stuart and A.V. Titov, Leptonic Dirac CP-violation Predictions from Residual Discrete Symmetries, Nucl. Phys. B 902 (2016) 1 [arXiv:1509.02502] [INSPIRE].

[53] I. Girardi, S.T. Petcov and A.V. Titov, Predictions for the Majorana CP-violation Phases in the Neutrino Mixing Matrix and Neutrinoless Double Beta Decay, Nucl. Phys. B 911 (2016) 754 [arXiv: 1605.04172] [INSPIRE].

[54] J.T. Penedo, S.T. Petcov and A.V. Titov, Neutrino Mixing and Leptonic CP-violation from $S_{4}$ Flavour and Generalised CP Symmetries, arXiv:1705.00309 [INSPIRE].

[55] T. Morozumi, H. Okane, H. Sakamoto, Y. Shimizu, K. Takagi and H. Umeeda, Phenomenological Aspects for Possible Vacua of Neutrino Flavor Model, arXiv:1707.04028 [INSPIRE].

[56] S.F. King, Littlest Seesaw, JHEP 02 (2016) 085 [arXiv: 1512.07531] [INSPIRE].

[57] P. Ballett, S.F. King, S. Pascoli, N.W. Prouse and T. Wang, Precision neutrino experiments vs the Littlest Seesaw, JHEP 03 (2017) 110 [arXiv:1612.01999] [INSPIRE].

[58] S.F. King and C. Luhn, Littlest Seesaw model from $S_{4} \times$ U(1), JHEP 09 (2016) 023 [arXiv: 1607.05276] [INSPIRE].

[59] Z. Maki, M. Nakagawa and S. Sakata, Remarks on the unified model of elementary particles, Prog. Theor. Phys. 28 (1962) 870 [inSPIRE].

[60] B. Pontecorvo, Neutrino Experiments and the Problem of Conservation of Leptonic Charge, Sov. Phys. JETP 26 (1968) 984 [INSPIRE].

[61] C. Jarlskog, Commutator of the Quark Mass Matrices in the Standard Electroweak Model and a Measure of Maximal CP-violation, Phys. Rev. Lett. 55 (1985) 1039 [InSPIRE]. 
[62] I. Esteban, M.C. Gonzalez-Garcia, M. Maltoni, I. Martinez-Soler and T. Schwetz, Updated fit to three neutrino mixing: exploring the accelerator-reactor complementarity, JHEP 01 (2017) 087 [arXiv: 1611.01514] [INSPIRE].

[63] P.F. de Salas, D.V. Forero, C.A. Ternes, M. Tortola and J.W.F. Valle, Status of neutrino oscillations 2017, arXiv: 1708.01186 [INSPIRE].

[64] J. Gehrlein, S.T. Petcov, M. Spinrath and A.V. Titov, Renormalisation Group Corrections to Neutrino Mixing Sum Rules, JHEP 11 (2016) 146 [arXiv:1608.08409] [INSPIRE].

[65] M. Fukugita and T. Yanagida, Baryogenesis Without Grand Unification, Phys. Lett. B 174 (1986) 45 [INSPIRE].

[66] C. Hagedorn, S.F. King and C. Luhn, A SUSY GUT of Flavour with $S_{4} \times \mathrm{SU}(5)$ to NLO, JHEP 06 (2010) 048 [arXiv: 1003.4249] [INSPIRE].

[67] S.F. King and C. Luhn, Neutrino Mass and Mixing with Discrete Symmetry, Rept. Prog. Phys. 76 (2013) 056201 [arXiv: 1301.1340] [INSPIRE]. 